\title{
BALANCED FRAMES: A USEFUL TOOL IN SIGNAL PROCESSING WITH GOOD PROPERTIES
}

\author{
SIGRID B. HEINEKEN ${ }^{1}$, PATRICIA M. MORILLAS ${ }^{2, *}$, AND PABLO TARAZAGA ${ }^{2,3}$ \\ ${ }^{1}$ Departamento de Matemática, FCEyN, Universidad de Buenos Aires, Pabellón I, Ciudad Universitaria, IMAS, \\ UBA-CONICET, C1428EGA C.A.B.A., Argentina \\ 2 Instituto de Matemática Aplicada San Luis, UNSL-CONICET, Ejército de los Andes 950, 5700 San Luis, \\ Argentina \\ 3 Departamento de Matemática, FCFMyN, UNSL, Ejército de los Andes 950, 5700 San Luis, Argentina
}

\begin{abstract}
So far there has not been paid attention to frames that are balanced, i.e. those frames which sum is zero. In this paper we consider balanced frames, and in particular balanced unit norm tight frames, in finite dimensional Hilbert spaces.

Here we discover various advantages of balanced unit norm tight frames in signal processing. They give an exact reconstruction in the presence of systematic errors in the transmitted coefficients, and are optimal when these coefficients are corrupted with noises that can have non-zero mean. Moreover, using balanced frames we can know that the transmitted coefficients were perturbed, and we also have an indication of the source of the error.

We analyze several properties of these types of frames. We define an equivalence relation in the set of the dual frames of a balanced frame, and use it to show that we can obtain all the duals from the balanced ones. We study the problem of finding the nearest balanced frame to a given frame, characterizing completely its existence and giving its expression. We introduce and study a concept of complement for balanced frames. Finally, we present many examples and methods for constructing balanced unit norm tight frames.
\end{abstract}

Key words: Balanced frames, unit norm tight frames, systematic errors, non-white noises, error detection, spherical designs.

AMS subject classification: Primary 42C15; Secondary 15A03, 15A60, 94A05, 94A12, $94 \mathrm{~A} 13$.

\section{Introduction}

A spanning set of vectors in a finite dimensional Hilbert space is called a frame. The redundancy of these spanning sets is the crucial property in their vast types of applications in many different areas of pure and applied mathematics and sciences, such as efficient representation of vectors and operators, signal processing, coding theory, communication theory, sampling theory, quantum information, and computing among others (see e.g. 11, 12, 24, 29, 34).

In this paper we study balanced frames, i.e. those frames which sum is zero, and several particular cases of them, especially balanced unit norm tight frames (see e.g. [5] for the concept of unit norm tight frame).

We show that although non balanced unit norm tight frames are optimal in many situations that appear in applications (see e.g. [5, 10, 11, 16, 34] and the references therein), balanced unit norm tight frames are even optimal in cases where the non balanced are not the best ones.

\footnotetext{
* Corresponding author. E-mail address: morillas.unsl@gmail.com E-mail addresses: sheinek@dm.uba.ar (S. B. Heineken), morillas.unsl@gmail.com (P. M. Morillas), patarazaga@hotmail.com (P. Tarazaga).
} 
In applications, a signal $f$ is usually represented by a sequence of numbers which are measurements of $f$. In frame theory, these measurements are expressed as inner products of $f$ with the elements of a frame, and will be called frame coefficients.

As we will explain in this work, the reconstructions using balanced frames are robust against systematic errors in the frame coefficients. Systematic errors can come from a wrong calibration of instruments, inexact methods of observation, or interference of the environment in the measurement, transmission or reception processes. A systematic error can be produced, for example, by the incorrect zeroing of an instrument. Another example are measurements by radar that can be systematically overestimated if we do not take into account the slowing down of the waves in the air. Systematic errors are not random, and cannot be reduced by taking the average of many readings. Considering this, it is important to highlight that balanced frames are immune to these type of errors. This means that in the presence of a systematic error in the frame coefficients, balanced frames still give the exact reconstruction.

In signal processing, the frame coefficients can be perturbed with additive noises. It has been shown [16] that if the mean of these noises is zero, the reconstruction of the signal with unit norm tight frames is optimal. We prove that if we use balanced unit norm tight frames, these noises can have a nonzero mean but the reconstruction is still optimal. Thus we can deal with noises of different sources. If the mean is non-zero we are under the presence of non-white noises. Nonzero mean noises appear naturally in certain applications. Digital watermarking is an application for which the zero mean assumption for the noises is not realistic 23. It is a useful tool for multimedia copyright protection, access control, annotation and authentication [27, 14, 25]. In certain cases such as median filtering, a standard signal processing method for denoising, the noises in the watermarking channel are additive with a non-zero mean.

Given a frame, each element of the Hilbert space can be expressed as linear combinations of the elements of the frame using the so called dual frames. As we will see, another advantage of balanced frames is that they are resilient against a perturbation of the dual frame by a constant vector, i.e. if we sum to each element of the dual frame a fixed vector, we still obtain a dual frame. We use this fact to define an equivalence relation in the set of dual frames of a given balanced frame and prove that all the dual frames can be obtained from the balanced ones.

We show that balanced frames are robust against one erasure, that is, they remain to be a frame if we delete any of its elements. The dual frames of these subfamilies are easy to obtain from the dual frames of the original family.

If we use a balanced frame the sum of the frame coefficients is always zero. So, if the transmitted numbers do not have zero sum we know that they were perturbed. Moreover, as we will explain, if we use balanced frames we can have an indication of when we are in the presence of a systematic error, of random additive noises or of other sources of perturbation as e.g. erasures.

In 8, 34 it is proved that real balanced unit norm tight frames are spherical 2-designs, a mathematical object applied in different areas. We want to point out that in contrast to what usually occurs in the context of spherical 2-designs, we are not necessarily interested in working with the minimum possible number of elements since, as observed before, from the point of view of frame theory redundancy is convenient for the applications. It can be seen in [6, 17 that balanced unit norm tight frames have advantages for sigma-delta quantization. In 13 tight frames are characterized using balanced sequences via diagram vectors. Balanced frames are mentioned in [34] in the definition of simple lift. But this concept has not so far been developed neither their multiple advantages noticed.

1.1. Contents. In Section 2, we briefly review frames.

In Section 3 we analyze the various advantages of balanced frames and balanced unit norm tight frames for applications which were mentioned before. 
In Section 4 we show that balanced frames and in particular balanced equal norm frames and balanced unit norm tight frames behave well, in the sense that they are invariant under various transformations. We find several characterizations of them and analyze properties of their dual frames.

In Section 5 we study the closest balanced frame to a given frame in the $\ell^{1}$ and $\ell^{2}$ norms. We give necessary and sufficient conditions for the closest balanced frame to exist, and for the case it exists we give its expression.

In Section 6 we introduce a concept of complement that is more suitable for balanced frames than the definition used so far for frames in general. Properties of this new notion are given.

In Section 7 we give many examples of balanced unit norm tight frames such as those corresponding to roots of unity in $\mathbb{R}^{2}$, certain types of harmonic frames, frames obtained from Hadamard matrices, partition frames and some that are spherical $t$-designs.

Finally, in Section 8, we present several explicit and painless methods for constructing balanced unit norm tight frames.

\section{Preliminaries}

In this section we recall some concepts of frame theory [11, 12, 24, 29, 34]. We refer to the mentioned works for more details. We begin introducing some notation.

2.1. Notation. Let $d, K \in \mathbb{N}$. Let $\mathbb{H}_{d}$ be a Hilbert space of dimension $d$ over a field $\mathbb{F}$ where $\mathbb{F}=\mathbb{R}$ or $\mathbb{F}=\mathbb{C}$. We write $\langle.,$.$\rangle and \|$.$\| for the inner product and the norm in \mathbb{H}_{d}$, respectively. Let $\mathcal{L}\left(\mathbb{H}_{d}, \mathbb{H}_{K}\right)$ be the space of linear transformations from $\mathbb{H}_{d}$ to $\mathbb{H}_{K}$ (we write $\mathcal{L}\left(\mathbb{H}_{d}\right)$ for $\mathcal{L}\left(\mathbb{H}_{d}, \mathbb{H}_{d}\right)$ ). Let $\mathcal{G} l\left(\mathbb{H}_{d}\right)\left(\mathcal{U}\left(\mathbb{H}_{d}\right)\right)$ be set of invertible (unitary) elements in $\mathcal{L}\left(\mathbb{H}_{d}\right)$. If $T \in \mathcal{L}\left(\mathbb{H}_{d}, \mathbb{H}_{K}\right)$, then $\operatorname{im}(T), \operatorname{ker}(T)$ and $T^{*}$ denote the range, the kernel and the adjoint of $T$, respectively. If $T \in \mathcal{L}\left(\mathbb{H}_{d}\right)$ and $\left(f_{k}\right)_{k=1}^{K}$ is a sequence in $\mathbb{H}_{d}$, we will write $T\left(f_{k}\right)_{k=1}^{K}$ for $\left(T f_{k}\right)_{k=1}^{K}$. The elements of $\mathbb{F}^{K}$ will be considered as column vectors. We write $e$ for the real vector which entries are all equal to 1 .

2.2. Frames. To a sequence $\mathcal{F}=\left(f_{k}\right)_{k=1}^{K}$ in $\mathbb{H}_{d}$ we associate the synthesis operator

the analysis operator

$$
T_{\mathcal{F}}: \mathbb{F}^{K} \rightarrow \mathbb{H}_{d}, T_{\mathcal{F}} c=\sum_{k=1}^{K} c_{k} f_{k},
$$

the frame operator

$$
T_{\mathcal{F}}^{*}: \mathbb{H}_{d} \rightarrow \mathbb{F}^{K}, T_{\mathcal{F}}^{*} f=\left(\left\langle f, f_{k}\right\rangle\right)_{k=1}^{K},
$$

and the Gram operator

$$
S_{\mathcal{F}}=T_{\mathcal{F}} T_{\mathcal{F}}^{*},
$$

$$
G_{\mathcal{F}}=T_{\mathcal{F}}^{*} T_{\mathcal{F}} .
$$

Definition 2.1. Let $\mathcal{F}=\left(f_{k}\right)_{k=1}^{K}$ be a sequence in $\mathbb{H}_{d}$. $\mathcal{F}$ is a frame for $\mathbb{H}_{d}$ if span $\mathcal{F}=\mathbb{H}_{d}$.

Proposition 2.2. Let $\mathcal{F}=\left(f_{k}\right)_{k=1}^{K}$ be a sequence in $\mathbb{H}_{d}$. The following assertions are equivalent:

(1) $\mathcal{F}$ is a frame for $\mathbb{H}_{d}$.

(2) $T_{\mathcal{F}}$ is onto.

(3) $T_{\mathcal{F}}^{*}$ is one to one.

(4) $S_{\mathcal{F}}$ is invertible.

(5) $\operatorname{rank}\left(G_{\mathcal{F}}\right)=d$.

(6) There exist $\alpha, \beta>0$ such that

$$
\alpha\|f\|^{2} \leq \sum_{k=1}^{K}\left|\left\langle f, f_{k}\right\rangle\right|^{2} \leq \beta\|f\|^{2} \text { for all } f \in \mathcal{H} .
$$


We call $\alpha$ and $\beta$ in (2.1) the frame bounds. The optimal lower frame bound is $\lambda_{\min }\left(S_{\mathcal{F}}\right)=$ $\left\|S_{\mathcal{F}}^{-1}\right\|^{-1}$ and the optimal upper frame bound is $\lambda_{\max }\left(S_{\mathcal{F}}\right)=\left\|S_{\mathcal{F}}\right\|=\left\|T_{\mathcal{F}}\right\|^{2}$ where $\lambda_{\min }\left(S_{\mathcal{F}}\right)$ and $\lambda_{\max }\left(S_{\mathcal{F}}\right)$ are the smallest and largest eigenvalues of $S_{\mathcal{F}}$, respectively.

Definition 2.3. Let $\mathcal{F}=\left(f_{k}\right)_{k=1}^{K}$ be a sequence in $\mathbb{H}_{d}$. We say that:

(1) $\mathcal{F}$ is balanced (B) if $\sum_{k=1}^{K} f_{k}=0$.

(2) $\mathcal{F}$ is real if $G_{\mathcal{F}}$ is a real matrix.

(3) $\mathcal{F}$ is equal-norm (EN) if $\left\|f_{k}\right\|=\left\|f_{k^{\prime}}\right\|$ for $k, k^{\prime}=1, \ldots, K$. $\mathcal{F}$ is unit norm (UN) if $\left\|f_{k}\right\|=1$ for $k=1, \ldots, K$.

(4) $\mathcal{F}$ is isogonal if $\mathcal{F}$ is EN and there exists an $a \in \mathbb{R}$ such that $\left\langle f_{k}, f_{l}\right\rangle=a$ for $k, l \in\{1, \ldots, K\}$, $k \neq l$.

Isogonal vectors appear in 28 in relation with the structure of soap films and bubbles. They are a particular case of equiangular frames [18, 33], i.e., EN frames for which there exists $a \in \mathbb{R}$ such that $\left|\left\langle f_{k}, f_{l}\right\rangle\right|=a$ for $k, l \in\{1, \ldots, K\}, k \neq l$. A unit norm frame is a (spherical) $m$-distance frame if the inner products between distinct vectors take $m$ real values 34 . A unit norm isogonal frame is a 1-distance frame. For the case $m=2$ see e.g. [4].

Definition 2.4. Let $\mathcal{F}=\left(f_{k}\right)_{k=1}^{K}$ be a frame for $\mathbb{H}_{d}$.

(1) $\mathcal{F}$ is an $\alpha$-tight frame $\left(\alpha\right.$-TF) if $S_{\mathcal{F}}=\alpha I . \mathcal{F}$ is a Parseval frame $(\mathrm{PF})$ if $S_{\mathcal{F}}=I$.

(2) $\mathcal{F}$ is maximally robust to erasures if every subset of $\mathcal{F}$ with $d$ elements is a basis for $\mathbb{H}_{d}$.

(3) $\mathcal{F}$ is a simplex frame if $G_{\mathcal{F}}=I-\frac{1}{K} e e^{t}$.

Maximally robust to erasures frames appeared first in 30. They are also known as generic frames [9] and full spark frames [1].

If $\mathbb{H}_{d}=\mathbb{R}^{d}$ and $\mathcal{F}$ is a simplex frame, then $\mathcal{F}$ corresponds to the $d+1$ vertices of the regular $d$-simplex in $\mathbb{R}^{d}$. We note that a 1 -simplex is a line segment, a 2 -simplex is a triangle, a 3 -simplex is a tetrahedron and a 4-simplex is a pentachoron or pentatope.

The following proposition collects some properties of frames.

Proposition 2.5. Let $\mathcal{F}=\left(f_{k}\right)_{k=1}^{K}$ be a frame for $\mathbb{H}_{d}$.

(1) If $\mathcal{F}$ is an $\alpha$-UNTF then $\alpha=\frac{K}{d}$.

(2) $\mathcal{F}$ is a UNPF if and only if $\mathcal{F}$ is an orthonormal basis.

(3) $\mathcal{F}$ is a PF with $K=d$ if and only if $\mathcal{F}$ is an orthonormal basis.

(4) $\mathcal{F}$ is an $\alpha-T F$ if and only if $\frac{1}{\alpha} \mathcal{F}$ is a Parseval frame.

(5) $\mathcal{F}$ is Parseval if and only if $G_{\mathcal{F}}$ is an orthogonal projection.

(6) $S_{\mathcal{F}}^{-1 / 2} \mathcal{F}$ is a PF for $\mathbb{H}_{d}$.

(7) If $\mathcal{F}$ is a simplex frame then $K=d+1$ and $\mathcal{F}$ is an isogonal PF.

(8) Let $\mathcal{W}$ be a subspace of $\mathbb{H}_{d}$ and $\pi_{\mathcal{W}}$ be the orthogonal projection onto $\mathcal{W}$. If $\mathcal{F}$ is an $\alpha-T F$ for $\mathbb{H}_{d}$ then $\pi_{\mathcal{W}} \mathcal{F}$ is an $\alpha-T F$ for $\mathcal{W}$.

Definition 2.6. Two frames $\mathcal{F}$ and $\mathcal{G}$ are complements of each other if the sum of the Gramians of $S_{\mathcal{F}}^{-1 / 2} \mathcal{F}$ and $S_{\mathcal{G}}^{-1 / 2} \mathcal{G}$ is the identity $I$.

The complement of a frame of $K$ vectors for a space of dimension $d$ is a frame of $K$ vectors for a space of dimension $K-d$.

Definition 2.7. Let $\mathcal{F}=\left(f_{k}\right)_{k=1}^{K}$ and $\mathcal{G}=\left(g_{k}\right)_{k=1}^{K}$ be frames for $\mathbb{H}_{d}$. Then $\mathcal{G}$ is a dual frame of $\mathcal{F}$ if the following reconstruction formula holds

or equivalently,

$$
f=\sum_{k=1}^{K}\left\langle f, f_{k}\right\rangle g_{k}, \text { for all } f \in \mathbb{H}_{d},
$$

$$
T_{\mathcal{G}} T_{\mathcal{F}}^{*}=I \text {. }
$$


Let $\mathcal{F}=\left(f_{k}\right)_{k=1}^{K}$ be a frame for $\mathbb{H}_{d}$. Then $\left(S_{\mathcal{F}}^{-1} f_{k}\right)_{k=1}^{K}$ is the canonical dual frame of $\mathcal{F}$. If $\mathcal{F}$ is an $\alpha$-tight frame for $\mathbb{H}_{d}$ we have the following reconstruction formula

$$
f=\frac{1}{\alpha} \sum_{k=1}^{K}\left\langle f, f_{k}\right\rangle f_{k}, \text { for all } f \in \mathbb{H}_{d}
$$

Proposition 2.8. Let $\mathcal{F}=\left(f_{k}\right)_{k=1}^{K}$ be a frame for $\mathbb{H}_{d}$ and $\mathcal{G}=\left(g_{k}\right)_{k=1}^{K}$ be a sequence in $\mathbb{H}_{d}$. The following assertions are equivalent:

(1) $\mathcal{G}$ is a dual frame of $\mathcal{F}$.

(2) $T_{\mathcal{G}}=S_{\mathcal{F}}^{-1} T_{\mathcal{F}}+R$ with $R \in \mathcal{L}\left(\mathbb{F}^{K}, \mathbb{H}_{d}\right)$ and $R T_{\mathcal{F}}^{*}=0$.

(3) $T_{\mathcal{G}}=S_{\mathcal{F}}^{-1} T_{\mathcal{F}}+W\left(I-T_{\mathcal{F}}^{*} S_{\mathcal{F}}^{-1} T_{\mathcal{F}}\right)$ with $W \in \mathcal{L}\left(\mathbb{F}^{K}, \mathbb{H}_{d}\right)$.

Note that a sequence $\left(f_{k}\right)_{k=1}^{K}$ in $\mathbb{H}_{d}$ is a BUNTF if and only if $\left(\sqrt{\frac{d}{K}} f_{k}\right)_{k=1}^{K}$ is a BENPF. In view of this, we will work either with BUNTFs or BENPFs according to convenience.

\section{Applications of BAlanCed frames}

In this section we describe various advantages of balanced frames and BUNTFs for applications. We will see that we can gain already very good properties assuming only balancedness, which is a condition that can be easily obtained. Note e.g. that if $\left(f_{k}\right)_{k=1}^{K}$ is a frame then $\left(f_{1}, \ldots, f_{K},-\sum_{k=1}^{K} f_{k}\right)$ is a balanced frame.

As we mentioned in the introduction, the measurements of a signal $f$, that in frame theory are expressed as inner products of $f$ with the elements of a frame, are used to represent it and will be called frame coefficients. In obtaining these measurements, or in the transmission or reception of them, different errors or erasures can occur.

3.1. Robustness of the reconstructions under systematic errors. Let $\mathcal{F}=\left(f_{k}\right)_{k=1}^{K}$ be a frame for $\mathbb{H}_{d}$. Any $f \in \mathbb{H}_{d}$ can be represented as a linear combination $f=\sum_{k=1}^{K} c_{k} f_{k}$. If $\mathcal{F}$ is balanced we can change the numbers $c_{k}$ by summing to each of them a constant $c$ and the reconstruction will still be the desired one, i.e., $f=\sum_{k=1}^{K}\left(c_{k}+c\right) f_{k}$. This situation occurs in the presence of systematic errors. The previous considerations show that the reconstruction using balanced frames is not affected by these type of errors. This is a very important fact, because repeating the readings numerous times and taking the average of them will not decrease systematic errors. Note that $c$ can vary with $f$, as it happens with the $c_{k}$, and it can also be random.

3.2. Reconstruction error bounds. Let $\mathcal{F}=\left(f_{k}\right)_{k=1}^{K}$ be a BF for $\mathbb{H}_{d}$. Assume that $\left(\left\langle f, f_{k}\right\rangle\right)_{k=1}^{K}$ is perturbed by the additive noises $\left(a_{k}\right)_{k=1}^{K}$, i.e. we have the sequence $\left(\left\langle f, f_{k}\right\rangle+a_{k}\right)_{k=1}^{K}$. Then the reconstruction $\hat{f}$ is

$$
\hat{f}=\sum_{k=1}^{K}\left(\left\langle f, f_{k}\right\rangle+a_{k}\right) S_{\mathcal{F}}^{-1} f_{k}=f+\sum_{k=1}^{K} a_{k} S_{\mathcal{F}}^{-1} f_{k}
$$

Since the frame is balanced we can give error bounds assuming that the noises are near a constant that is not necessarily equal to zero:

Proposition 3.1. Let $f \in \mathbb{H}_{d}$ and $\left(f_{k}\right)_{k=1}^{K}$ be a balanced frame for $\mathbb{H}_{d}$. The following statements about the norm of the reconstruction error hold:

(1) Suppose that there exists $\mu$ such that $\left|a_{k}-\mu\right|<\epsilon$ for each $k=1, \ldots, K$. Let $\lambda_{\max }=$ $\lambda_{1} \geq \lambda_{2} \geq \ldots \geq \lambda_{d}=\lambda_{\min }>0$ be the eigenvalues of $S_{\mathcal{F}}$. Then $\|f-\hat{f}\|<\sqrt{K / \lambda_{\min }} \epsilon$. Furthermore, if $\left(f_{k}\right)_{k=1}^{K}$ is a BUNTF for $\mathbb{H}_{d}$ we can assert that $\|f-\hat{f}\|<\sqrt{d} \epsilon$ and $\|f-\hat{f}\|_{\infty}<d \epsilon$.

(2) Assume that there exists $\mu$ such that $\left(\sum_{k=1}^{K}\left|a_{k}-\mu\right|^{2}\right)^{1 / 2}<\epsilon$. If $\left(f_{k}\right)_{k=1}^{K}$ is a BUNTF for $\mathbb{H}_{d}$ then $\|f-\hat{f}\|<\sqrt{d / K} \epsilon$. 
Proof. For the first inequality we can argue similarly as in the proof of [17, Proposition 2.1]. Consider the canonical dual of $\mathcal{F}, \mathcal{G}=S_{\mathcal{F}}^{-1} \mathcal{F}$. Then $T_{\mathcal{G}}=S_{\mathcal{F}}^{-1} T_{\mathcal{F}}$. The reconstruction error is $f-\hat{f}=\sum_{k=1}^{K} a_{k} S_{\mathcal{F}}$
where $y=\left(a_{1}-\mu, \ldots, a_{K}-\mu\right)^{t}$. Hence

$$
\|f-\hat{f}\|^{2}=y^{t} T_{\mathcal{G}}^{t} T_{\mathcal{G}} y \leq \rho\left(T_{\mathcal{G}}^{t} T_{\mathcal{G}}\right)\|y\|^{2}<\rho\left(T_{\mathcal{G}}^{t} T_{\mathcal{G}}\right) K \epsilon^{2}
$$

where $\rho(\cdot)$ is the spectral radius. But $\rho\left(T_{\mathcal{G}}^{t} T_{\mathcal{G}}\right)=\rho\left(T_{\mathcal{G}} T_{\mathcal{G}}^{t}\right)=\rho\left(S_{\mathcal{F}}^{-1}\right)=\frac{1}{\lambda_{\min }}$, so the first part of (1) follows.

Now assume that $\left(f_{k}\right)_{k=1}^{K}$ is a BUNTF for $\mathbb{H}_{d}$. In this case $\lambda_{1}=\lambda_{2}=\ldots=\lambda_{d}=\lambda_{\text {min }}=\frac{K}{d}$, so from the previous result $\|f-\hat{f}\|<\sqrt{d} \epsilon$. We also have

$$
\|f-\hat{f}\|_{\infty}=\left\|\sum_{k=1}^{K}\left(a_{k}-\mu\right) \frac{d}{K} f_{k}\right\|_{\infty} \leq \frac{d}{K} \sum_{k=1}^{K} \mid a_{k}-\mu\|\| f_{k} \|_{\infty}<\epsilon d .
$$

For (2) observe that $\|f-\hat{f}\|=\left\|\sum_{k=1}^{K}\left(a_{k}-\mu\right) \frac{d}{K} f_{k}\right\| \leq \frac{d}{K} \sqrt{\frac{K}{d}}\left(\sum_{k=1}^{K}\left|a_{k}-\mu\right|^{2}\right)^{1 / 2}<\sqrt{\frac{d}{K}} \epsilon$.

3.3. Presence of random additive noises without the zero mean assumption. We can analyze the behavior of the reconstruction error using a statistical model for noise. Let $\mathcal{F}=\left(f_{k}\right)_{k=1}^{K}$ be a BUNF for $\mathbb{R}^{d}$ with frame bounds $\alpha, \beta$.

Assume now that $\left(\left\langle f, f_{k}\right\rangle\right)_{k=1}^{K}$ is perturbed with additive noises $\left(\eta_{k}\right)_{k=1}^{K}$, and that each noise $\eta_{k}$ is a random variable with mean $E\left[\eta_{k}\right]=\mu$ and variance $E\left[\left(\eta_{k}-\mu\right)^{2}\right]=\sigma^{2}$. Suppose also that the noises $\eta_{k}$ and $\eta_{l}$ are uncorrelated for $k \neq l$, i.e. $\operatorname{cov}\left(\eta_{k}, \eta_{l}\right)=E\left[\left(\eta_{k}-\mu\right)\left(\eta_{l}-\mu\right)\right]=\delta_{k, l} \sigma^{2}$ for each $k, l$. As before, the receiver will reconstruct the signal as

$$
\hat{f}=\sum_{k=1}^{K}\left(\left\langle f, f_{k}\right\rangle+\eta_{k}\right) S_{\mathcal{F}}^{-1} f_{k}=f+\sum_{k=1}^{K} \eta_{k} S_{\mathcal{F}}^{-1} f_{k} .
$$

The advantage of considering the balanced case in what follows is that the mean of the noises is not required to be zero, an assumption needed for the non balanced case which has been considered so far in the literature.

The mean square error is $M S E:=\frac{1}{d} E\left[\left\|\sum_{k=1}^{K} \eta_{k} S_{\mathcal{F}}^{-1} f_{k}\right\|^{2}\right]$. Since $\mathcal{F}$ is balanced, we can write

$$
M S E=\frac{1}{d} E\left[\left\|\sum_{k=1}^{K}\left(\eta_{k}-\mu\right) S_{\mathcal{F}}^{-1} f_{k}\right\|^{2}\right] .
$$

The assumptions on the noises lead to

$$
M S E=\frac{1}{d} \sigma^{2} \sum_{k=1}^{K}\left\|S_{\mathcal{F}}^{-1} f_{k}\right\|^{2} .
$$

So,

$$
\frac{K \sigma^{2}}{d \beta^{2}} \leq M S E \leq \frac{K \sigma^{2}}{d \alpha^{2}} .
$$

If $d$ and $K$ are fixed it can be proved, as in [16. Theorem 3.1] but now without assuming $\mu=0$, that the MSE is minimal if and only if the frame is tight and that in this case $M S E=\frac{d}{K} \sigma^{2}$.

Sometimes the reconstruction is done using the orthogonal projection of $\left(\left\langle f, f_{k}\right\rangle+\eta_{k}\right)_{k=1}^{K}$ onto $R\left(T_{\mathcal{F}}^{*}\right)$ given by $p=T_{\mathcal{F}}^{*} f+T_{\mathcal{F}}^{*} S_{\mathcal{F}}^{-1} T_{\mathcal{F}}\left(\eta_{k}\right)_{k=1}^{K}$. Since the frame is balanced,

$$
p=T_{\mathcal{F}}^{*} f+T_{\mathcal{F}}^{*} S_{\mathcal{F}}^{-1} T_{\mathcal{F}}\left(\eta_{k}-\mu e\right)_{k=1}^{K}=T_{\mathcal{F}}^{*} f+\widetilde{p} .
$$

So, as in [12, Section 8.5] but again without assuming $\mu=0$, it can be proved that,

$$
\frac{\sigma^{2}}{\beta} \leq E\left[|\widetilde{p}(k)|^{2}\right] \leq \frac{\sigma^{2}}{\alpha}
$$

where the equality holds if $\left(f_{k}\right)_{k=1}^{K}$ is a tight frame. In this case, $E\left[|\widetilde{p}(k)|^{2}\right]=\frac{d}{K} \sigma^{2}$.

Note that when considering BUNTFs, if the number of elements of the frame increases (higher redundancy) both the MSE and the mean of $|\widetilde{p}(k)|^{2}$ decrease. This shows the advantage of using redundant BUNTFs.

3.4. Resilience of the dual frames against fixed perturbations. Let $\left(f_{k}\right)_{k=1}^{K},\left(g_{k}\right)_{k=1}^{K}$ be sequences in $\mathbb{H}_{d}$ where $\left(f_{k}\right)_{k=1}^{K}$ is balanced. Then $\sum_{k=1}^{K}\left\langle f, g_{k}\right\rangle f_{k}=\sum_{k=1}^{K}\left\langle f,\left(g_{k}+g\right)\right\rangle f_{k}$ for each $g \in \mathbb{H}_{d}$. As a consequence of this we obtain:

Proposition 3.2. If $\mathcal{F}$ is a balanced frame for $\mathbb{H}_{d}$ and $\mathcal{G}=\left(g_{k}\right)_{k=1}^{K}$ is a dual frame of $\mathcal{F}$, then $\left(g_{k}+g\right)_{k=1}^{K}$ is also a dual frame of $\mathcal{F}$ for each $g \in \mathbb{H}_{d}$. 
Proposition 3.2 says that for a balanced frame $\mathcal{F}$ the reconstruction is not altered if we use a dual which is perturbed by a fixed vector, and can also be used to define an equivalence relation in the set of dual frames of $\mathcal{F}$.

Definition 3.3. Let $\mathcal{F}$ be a balanced frame for $\mathbb{H}_{d}$. We say that two dual frames $\mathcal{G}=\left(g_{k}\right)_{k=1}^{K}$ and $\widetilde{\mathcal{G}}=\left(\widetilde{g}_{k}\right)_{k=1}^{K}$ of $\mathcal{F}$ are equivalent if there exists $g \in \mathbb{H}_{d}$ such that $\widetilde{g}_{k}=g_{k}+g$ for each $k=1, \ldots, K$.

It is clear that if there exists a balanced frame in an equivalence class, it is the unique balanced one in this class. Let $[\mathcal{G}]=\left\{\left(g_{k}+g\right)_{k=1}^{K}: g \in \mathbb{H}_{d}\right\}$ be the equivalence class of the dual frame $\mathcal{G}$ of $\mathcal{F}$. If $\mathcal{G}$ is not balanced, the dual frame $\left(g_{k}-\frac{1}{K} T_{\mathcal{G}} e\right)_{k=1}^{K}$ is equivalent to $\mathcal{G}$ and is balanced. This shows that each of the equivalence classes contains a unique dual frame which is balanced and can be considered as the representative of the class. Thus, in order to obtain all the dual frames of $\mathcal{F}$, we only need to compute those that are balanced, the others will be in their equivalence classes.

3.5. Presence of erasures. When some of the frame coefficients are no longer accessible after the transmission, we say that an erasure occurs.

Part (1) of the following proposition says that if one of the frame coefficients is deleted (or is set equal to zero) we can still recover $f$ exactly. It also says that a balanced frame $\left(f_{k}\right)_{k=1}^{K}$ remains to be a frame if we delete one of its elements. Joining both parts of the proposition we have a characterization of balanced frames.

Proposition 3.4. Let $\left(f_{k}\right)_{k=1}^{K}$ be a frame for $\mathbb{H}_{d}$ and $\left(\widetilde{f}_{k}\right)_{k=1}^{K}$ be one of its duals. The following assertions holds:

(1) If $\left(f_{k}\right)_{k=1}^{K}$ is balanced, then for each $l \in\{1, \ldots, K\},\left(f_{k}\right)_{k=1, k \neq l}^{K}$ and $\left(\widetilde{f}_{k}-\widetilde{f}_{l}\right)_{k=1, k \neq l}^{K}$ are dual frames.

(2) If there exists $l \in\{1, \ldots, K\}$ such that $\tilde{f}_{l} \neq 0$, and $\left(f_{k}\right)_{k=1, k \neq l}^{K}$ and $\left(\widetilde{f}_{k}-\widetilde{f}_{l}\right)_{k=1, k \neq l}^{K}$ are dual frames, then $\left(f_{k}\right)_{k=1}^{K}$ is balanced.

Proof. (1) Suppose that $\left(f_{k}\right)_{k=1}^{K}$ is balanced. Let $l \in\{1, \ldots, K\}$. Then for each $f \in \mathbb{H}_{d}$, $f=\sum_{k=1}^{K}\left\langle f, f_{k}\right\rangle \widetilde{f}_{k}=\sum_{k=1}^{K}\left\langle f, f_{k}\right\rangle\left(\widetilde{f}_{k}-\widetilde{f}_{l}\right)=\sum_{k=1, k \neq l}^{K}\left\langle f, f_{k}\right\rangle\left(\widetilde{f}_{k}-\widetilde{f}_{l}\right)$.

This last expression says that $\left(f_{k}\right)_{k=1, k \neq l}^{K}$ and $\left(\widetilde{f}_{k}-\widetilde{f}_{l}\right)_{k=1, k \neq l}^{K}$ are dual frames.

(2) Suppose that $l \in\{1, \ldots, K\}$ is such that $\tilde{f}_{l} \neq 0$ and that $\left(f_{k}\right)_{k=1, k \neq l}^{K}$ and $\left(\tilde{f}_{k}-\widetilde{f}_{l}\right)_{k=1, k \neq l}^{K}$ are dual frames. If $f \in \mathbb{H}_{d}$, then

$$
f=\sum_{k=1, k \neq l}^{K}\left\langle f, \widetilde{f}_{k}-\widetilde{f}_{l}\right\rangle f_{k}=\sum_{k=1}^{K}\left\langle f, \widetilde{f}_{k}-\widetilde{f}_{l}\right\rangle f_{k}=f-\left\langle f, \widetilde{f}_{l}\right\rangle \sum_{k=1}^{K} f_{k} .
$$

Taking $f=\tilde{f}_{l} \neq 0$, we obtain $\sum_{k=1}^{K} f_{k}=0$.

3.6. Error detection. If $\left(f_{k}\right)_{k=1}^{K}$ is a balanced frame, then $\sum_{k=1}^{K}\left\langle f, f_{k}\right\rangle=0$. So if the transmitted numbers $\left(c_{k}\right)_{k=1}^{K}$ satisfy $\sum_{k=1}^{K} c_{k} \neq 0$, we know that $\left(c_{k}\right)_{k=1}^{K}$ comes from a perturbation of the frame coefficients $\left(\left\langle f, f_{k}\right\rangle\right)_{k=1}^{K}$. In this way we can easily detect the presence of a problem.

Furthermore, using balanced frames we can have a hint about the source of the error. If we are in the presence of a systematic error i.e. $c_{k}=\left\langle f, f_{k}\right\rangle+c$ for some constant $c$, then $\sum_{k=1}^{K} c_{k}=K c$ independently of the signal $f$. In this case, although we can know the error, it is not necessary to correct it because the reconstruction with a balanced dual frame will be the desired one. If the perturbation is due to random additive noises $\left(\eta_{k}\right)_{k=1}^{K}$ with $\left|\eta_{k}-\eta\right| \leq \sigma$ for each $k$, then $c_{k}=\left\langle f, f_{k}\right\rangle+\eta_{k}$ and $\sum_{k=1}^{K} c_{k}=\sum_{k=1}^{K} \eta_{k}$ fluctuates without any apparent pattern between two fixed values, also independently of the signal. If instead the sum of the transmitted numbers is non zero and varies with the signal, we can suspect that the error arises from other sources. For example, assume that erasures occur, i.e. we only receive $\left(\left\langle f, f_{k}\right\rangle\right)_{k \in I}$ where $I$ is a proper subset of $\{1, \ldots, K\}$. In this case $\sum_{k \in I}\left\langle f, f_{k}\right\rangle$ generally varies with the signal $f$. 
3.7. BUNTFs for $\mathbb{R}^{d}$ and real spherical 2-designs. Real spherical t-designs appear in relation with cubature formulas on the sphere in $\mathbb{R}^{d}\left[3\right.$. They are sets of points on the unit sphere $\mathbb{S}^{d-1}$ of $\mathbb{R}^{d}$ such that the integral on $\mathbb{S}^{d-1}$ of any homogeneous polynomial of total degree less than or equal to $t$ in $d$ variables is equal to the mean of the values of the polynomial over these points. In other words, they approximate the unit sphere in the sense that computing the average of these polynomials only over these sets of points is identical to taking the average over the entire unit sphere. The following result can be found in different forms e.g. in [8, 32] (see also [34).

Proposition 3.5. A sequence $\left(f_{k}\right)_{k=1}^{K}$ of unit vectors in $\mathbb{R}^{d}$ is a spherical 2-design if and only if it is a BUNTF for $\mathbb{R}^{d}$.

Spherical $t$-designs are used in approximation theory, in numerical interpolation, integration, and regularized least squares approximation. They have connections with many areas of mathematics such as analysis and statistics (in particular with orthogonal polynomials and moment problems), algebraic combinatorics (association schemes, design theory, coding theory), group theory (spherical designs which are orbits of a finite group in the real orthogonal group $O(n)$ ), number theory (designs that are shells of Euclidean lattices are related with modular forms and the Lehmer's conjecture about the zeros of the Ramanujan $r$ function), geometry (sphere packing problems) and optimization (Delsarte's linear programming method).

3.8. Balanced sequences and tight frames. There is a strong connection between balanced sequences and tight frames via diagram vectors. Diagram vectors can be used for determining whether a frame for $\mathbb{R}^{2}$ is tight or not [19. This notion has been extended to $\mathbb{F}^{d}$ in 13 (we refer the reader to this paper for the definition of diagram vectors). By [13, Proposition 3.6] and Proposition 4.4 below, we have:

Proposition 3.6. Let $c_{1}, \ldots, c_{K}$ be nonnegative numbers, which are not all zero. Let $\left(f_{k}\right)_{k=1}^{K}$ in $\mathbb{F}^{d}$ and let $\left(\widetilde{f}_{k}\right)_{k=1}^{K}$ in $\mathbb{F}^{d}$ the corresponding diagram vectors. Then $\left(c_{k} f_{k}\right)_{k=1}^{K}$ is a tight frame for $\mathbb{F}^{d}$ if and only if $\left(c_{k}^{2} \widetilde{f}_{k}\right)_{k=1}^{K}$ is balanced.

This result shows that balanced sequences are useful in the study of tight frames.

\section{Properties}

In this section we study properties of BFs and in particular of BPFs and BUNTFs. We consider their behavior under transformations, give several characterizations and analyze duality.

4.1. Invariance under certain transformations. Given a frame, it is important which properties are preserved under certain transformations. The following are analogous to those presented in [30, here we analyze them regarding balancedness.

Proposition 4.1. (1) Let $A \in \mathcal{G l} l\left(\mathbb{H}_{d}\right)$ and $B \in \mathcal{G l} l\left(\mathbb{F}^{K}\right)$. If $B e=e$, then $\mathcal{F}$ is a balanced frame if and only if $A \mathcal{F} B$ is a balanced frame.

(2) Let $a \neq 0, U \in \mathcal{U}\left(\mathbb{H}_{d}\right)$ and $V \in \mathcal{U}\left(\mathbb{F}^{K}\right)$ such that $V e=e$. Then $a U \mathcal{F} V$ is a BTF if and only if $\mathcal{F}$ is a BTF.

(3) Let $a \neq 0, U \in \mathcal{U}\left(\mathbb{H}_{d}\right)$. Then $a U \mathcal{F}(U \mathcal{F})$ is a BENF (BUNF) if and only if $\mathcal{F}$ is a BENF (BUNF).

(4) Let $A \in \mathcal{G}\left(\mathbb{H}_{d}\right)$. Then $\mathcal{F}$ is a maximally robust to erasures $B F$ if and only if $A \mathcal{F}$ is a maximally robust to erasures $B F$.

(5) $\mathcal{F}$ is a BUNTF if and only if $\overline{\mathcal{F}}$ is a BUNTF.

(6) Let $\mathcal{W}$ be a subspace of $\mathbb{H}_{d}$ and $\pi_{\mathcal{W}}$ be the orthogonal projection onto $\mathcal{W}$. If $\mathcal{F}$ is an $\alpha$-BTF for $\mathbb{H}_{d}$ then $\pi_{\mathcal{W}} \mathcal{F}$ is an $\alpha$-BTF for $\mathcal{W}$. 
(7) Let $A \in \mathcal{L}\left(\mathbb{H}_{d}, \mathbb{H}_{n}\right)$ be an isometry, i.e., $A^{*} A=I$, then $\mathcal{F}$ is an $\alpha$-BUNTF for $\mathbb{H}_{d}$ if and only if $A \mathcal{F}$ is an $\alpha$-BUNTF for $\mathbb{H}_{n}$.

Proof. In each case, the proof follows straightforward from the definitions. To illustrate we show (1):

If $A \in \mathcal{L}\left(\mathbb{H}_{d}\right)$ is injective and $B \in \mathcal{L}\left(\mathbb{F}^{K}\right)$ is such that $B e=e$, then $T_{\mathcal{F}} e=0$ if and only if $A T_{\mathcal{F}} B e=0$. Moreover, if $A$ and $B$ are invertible, $T_{\mathcal{F}}$ is onto if and only if $A T_{\mathcal{F}} B$ is onto.

In view of Proposition 4.1, we define an equivalence relation:

Definition 4.2. Two frames $\mathcal{F}$ and $\mathcal{G}$ for $\mathbb{H}_{d}$ are unitary equivalent if and only if there exists a unitary operator $U \in L(\mathcal{H})$ such that $\mathcal{G}=U \mathcal{F}$.

In the previous equivalence relation, the permutation or numbering of the elements of $\mathcal{F}$ or $\mathcal{G}$ will not be considered. Two PFs are unitary equivalent if and only if they have the same Gram matrix [34, Corollary 2.1.].

As a consequence of Proposition 4.1 and Proposition 2.5.

Corollary 4.3. Let $\mathcal{F}$ be a frame for $\mathbb{H}_{d}$. The following assertions are equivalent:

(1) $\mathcal{F}$ is a $B F$ for $\mathbb{H}_{d}$.

(2) $S_{\mathcal{F}}^{-1} \mathcal{F}$ is a $B F$ for $\mathbb{H}_{d}$.

(3) $S_{\mathcal{F}}^{-1 / 2} \mathcal{F}$ is a BPF for $\mathbb{H}_{d}$.

4.2. Some characterizations. The following proposition gives several equivalences for a sequence to be balanced.

Proposition 4.4. Let $\mathcal{F}=\left(f_{k}\right)_{k=1}^{K}$ be a sequence in $\mathbb{H}_{d}$. The following assertions are equivalent:

(1) $\mathcal{F}$ is balanced.

(2) $T_{\mathcal{F}} e=0$.

(3) $G_{\mathcal{F}} e=0$.

(4) $\sum_{k=1}^{K}\left\langle f_{l}, f_{k}\right\rangle=0$ for each $l \in\{1, \ldots, K\}$.

(5) $\sum_{k, l=1}^{K}\left\langle f_{k}, f_{l}\right\rangle=0$.

(6) $\sum_{k=1}^{K}\left\langle f, f_{k}\right\rangle=0$ for each $f \in \mathbb{H}_{d}$.

(7) $\sum_{k=1}^{K}\left\|f-f_{k}\right\|^{2}=\sum_{k=1}^{K}\left\|f_{k}\right\|^{2}+K\|f\|^{2}$ for each $f \in \mathbb{H}_{d}$.

(8) $\sum_{k=1, k \neq l}^{K}\left\|f_{l}-f_{k}\right\|^{2}=\sum_{k=1}^{K}\left\|f_{k}\right\|^{2}+K\left\|f_{l}\right\|^{2}$ for each $l \in\{1, \ldots, K\}$.

Proof. Taking into account the definition of balanced sequences and that $\operatorname{ker}\left(T_{\mathcal{F}}\right)=\operatorname{ker}\left(G_{\mathcal{F}}\right)$, $(1) \Leftrightarrow(2) \Leftrightarrow(3)$ follows immediately.

Considering the entries of the matrix $G_{\mathcal{F}}$, it is immediate that $(3) \Leftrightarrow(4)$.

Observe that $\sum_{k, l=1}^{K}\left\langle f_{k}, f_{l}\right\rangle=\left\|T_{\mathcal{F}} e\right\|^{2}$. Thus we have $(2) \Leftrightarrow(5)$.

For $(1) \Leftrightarrow(6)$ we note that $\sum_{k=1}^{K} f_{k}=0$ if and only if $\left\langle f, \sum_{k=1}^{K} f_{k}\right\rangle=0$ for each $f \in \mathbb{H}_{d}$.

(6) $\Leftrightarrow$ (7) follows from $\left\|f-f_{k}\right\|^{2}=\left\|f_{k}\right\|^{2}-2 \operatorname{Re}\left(\left\langle f, f_{k}\right\rangle\right)+\|f\|^{2}$ and $\left\|i f-f_{k}\right\|^{2}=\left\|f_{k}\right\|^{2}+$ $2 \operatorname{Im}\left(\left\langle f, f_{k}\right\rangle\right)+\|f\|^{2}$. Similarly it can be proved $(4) \Leftrightarrow(8)$.

From Proposition 4.4 we can obtain the next well-known basic result about simplex frames:

Corollary 4.5. If $\mathcal{F}$ is a simplex frame then $\mathcal{F}$ is balanced.

There exists a bijective correspondence between the BUNTFs for $\mathbb{H}_{d}$ and the BUNTFs for its dual space. This is a consequence of the following result which follows from the Riesz representation theorem and Proposition 4.4 
Corollary 4.6. $\mathcal{F}=\left(f_{k}\right)_{k=1}^{K}$ is a BUNTF for $\mathbb{H}_{d}$ if and only if $\left(\left\langle., f_{k}\right\rangle\right)_{k=1}^{K}$ is a BUNTF for the dual space $\mathbb{H}_{d}^{*}$.

Proposition 3.4 says that a BF is one-robust to erasures. This suggests the following version of [10. Corollary 5.1]:

Proposition 4.7. Let $\left(e_{k}\right)_{k=1}^{K}$ be an orthonormal basis for $\mathbb{H}_{K}$ and $\pi_{\mathcal{W}}$ be the an orthogonal projection onto a subspace $\mathcal{W}$ of $\mathbb{H}_{K}$. The following are equivalent:

(1) $\left(\pi_{\mathcal{W}} e_{k}\right)_{k=1}^{K}$ is a balanced Parseval frame for $\mathcal{W}$.

(2) $\sum_{k=1}^{K} e_{k} \in \mathcal{W}^{\perp}$.

(3) There exists $f \in \mathcal{W}^{\perp}$ such that $\left\langle f, e_{k}\right\rangle=1$ for each $k=1, \ldots, K$.

Proof. By Proposition 2.5. $\left(\pi_{\mathcal{W}} e_{k}\right)_{k=1}^{K}$ is a Parseval frame for $\mathcal{W}$.

$(1) \Rightarrow(2)$ Since $\left(\pi_{\mathcal{W}} e_{k}\right)_{k=1}^{K}$ is balanced, $\pi_{\mathcal{W}} \sum_{k=1}^{K} e_{k}=0$. This shows that $\sum_{k=1}^{K} e_{k} \in \mathcal{W}^{\perp}$.

$(2) \Rightarrow(3)$ Take $f=\sum_{k=1}^{K} e_{k}$.

$(3) \Rightarrow(1)$ Let $f \in \mathcal{W}^{\perp}$ such that $\left\langle f, e_{k}\right\rangle=1$ for each $k=1, \ldots, K$. Then $\sum_{k=1}^{K} \pi_{\mathcal{W}} e_{k}=$ $\pi_{\mathcal{W}} \sum_{k=1}^{K}\left\langle f, e_{k}\right\rangle e_{k}=\pi_{\mathcal{W}} f=0$.

We have the following versions of the Naimark characterization for BPFs:

Theorem 4.8. A sequence $\left(f_{k}\right)_{k=1}^{K}$ in $\mathbb{H}_{d}$ is a BPF for $\mathbb{H}_{d}$ if and only if there is a larger Hilbert space $\mathbb{H}_{K} \supseteq \mathbb{H}_{d}$ and an orthonormal basis $\left(g_{k}\right)_{k=1}^{K}$ for $\mathbb{H}_{K}$ satisfying $\sum_{k=1}^{K} g_{k} \in \mathbb{H}_{d}^{\perp}$ so that $f_{k}=\pi_{\mathbb{H}_{d}} g_{k}$ for each $k=1, \ldots, K$.

Proof. Any Parseval frame $\mathcal{F}=\left(f_{k}\right)_{k=1}^{K}$ for $\mathbb{H}_{d}$ is unitary equivalent to $\left(G_{\mathcal{F}} e_{k}\right)_{k=1}^{K}$ where $\left(e_{k}\right)_{k=1}^{K}$ is the standard basis of $\mathbb{F}^{K}$ (see [34, Theorem 2.2.]). In this case, $G_{\mathcal{F}}$ is the orthogonal projection onto $\operatorname{im}\left(G_{\mathcal{F}}\right)$ that has dimension $d$. If $\mathcal{F}$ is also balanced then, by Proposition 4.4 $e=\sum_{k=1}^{K} e_{k} \in$ $\operatorname{im}\left(G_{\mathcal{F}}\right)^{\perp}$. Let $U \in L\left(\operatorname{im}\left(G_{\mathcal{F}}\right), \mathbb{H}_{d}\right)$ unitary such that $f_{k}=U G_{\mathcal{F}} e_{k}$ for each $k=1, \ldots, K$. Let $\mathbb{H}_{K}$ such that $\mathbb{H}_{K} \supseteq \mathbb{H}_{d}$ and $\mathbb{H}_{K}=\mathbb{H}_{d} \oplus \mathbb{H}_{d}^{\perp}$. Let $\widetilde{U} \in L\left(\mathbb{F}^{K}, \mathbb{H}_{K}\right)$ unitary such that $\widetilde{U}_{\mid \operatorname{im}\left(G_{\mathcal{F}}\right)}=U$. Let $g_{k}=\widetilde{U} e_{k}$ for each $k=1, \ldots, K$. Then $\pi_{H_{d}}=\widetilde{U} G_{\mathcal{F}} \widetilde{U}^{*},\left(g_{k}\right)_{k=1}^{K}$ is an orthonormal basis for $\mathbb{H}_{K}, \sum_{k=1}^{K} g_{k} \in \mathbb{H}_{d}^{\perp}$ and $f_{k}=\pi_{H_{d}} g_{k}$ for each $k=1, \ldots, K$.

Remark 4.9. The previous proof is constructive. The theorem follows also from Proposition 4.7 and Naimark's theorem [1].

Theorem 4.10. A sequence $\left(f_{k}\right)_{k=1}^{K}$ in $\mathbb{H}_{d}$ is a BPF for $\mathbb{H}_{d}$ if and only if there is a larger Hilbert space $\mathbb{H}_{K-1} \supseteq \mathbb{H}_{d}$ and a simplex frame $\left(g_{k}\right)_{k=1}^{K}$ for $\mathbb{H}_{K-1}$ so that $f_{k}=\pi_{\mathbb{H}_{d}} g_{k}$ for each $k=1, \ldots, K$.

Proof. Let $\mathcal{F}=\left(f_{k}\right)_{k=1}^{K}$ be a balanced Parseval frame for $\mathbb{H}_{d}$. Similar to the proof of Theorem 4.8, $\mathcal{F}$ is unitary equivalent to $\left(G_{\mathcal{F}}\left(e_{k}-\frac{1}{K} e\right)\right)_{k=1}^{K}$ where $\left(e_{k}\right)_{k=1}^{K}$ is the standard basis of $\mathbb{F}^{K}$. The sequence $\left(e_{k}-\frac{1}{K} e\right)_{k=1}^{K}$ is a simplex frame for $\operatorname{span}\{e\}^{\perp}$ that has dimension $K-1$. The rest follows as in proof of Theorem 4.8

Proposition 4.1 yields a decomposition of BUNTFs:

Proposition 4.11. Let $\left(f_{k}\right)_{k=1}^{K}$ be a sequence in $\mathbb{H}_{d}$ and $I \subseteq\{1, \ldots, K\}$ such that $f_{k} \perp f_{l}=0$ for $k \in I, l \in I^{c}$. Let $\mathcal{W}:=\operatorname{span}\left(f_{k}\right)_{k \in I}$. Then $\left(f_{k}\right)_{k=1}^{K}$ is BUNTF for $\mathbb{H}_{d}$ if and only if $\left(f_{k}\right)_{k \in I}$ is a $B U N T F$ for $\mathcal{W}$ and $\left(f_{k}\right)_{k \in I^{c}}$ is a BUNTF for $\mathcal{W}^{\perp}$.

The frame graph (or correlation network) of a sequence $\left(f_{k}\right)_{k=1}^{K}$ in $\mathbb{H}_{d}$ is the graph with vertices $\left(f_{k}\right)_{k=1}^{K}$ and an edge between $f_{k}$ and $f_{k^{\prime}}, k \neq k^{\prime}$, if and only if $\left\langle f_{k}, f_{k^{\prime}}\right\rangle \neq 0$ [34]. Each frame can be uniquely decomposed into a union of frames for orthogonal subspaces, each corresponding to the vertices of a connected component of the frame graph. Proposition 4.11 gives a characterization of BUNTFs in terms of the cycles in its frame graph: 
Theorem 4.12. A sequence $\left(f_{k}\right)_{k=1}^{K}$ in $\mathbb{H}_{d}$ is a BUNTF if and only if the vertices of each of the connected components in its frame graph is a BUNTF for its span.

4.3. Duals of a balanced frame. As was explained in section 3.4, in order to obtain the duals of a balanced frame it is sufficient to consider the balanced ones. Proposition 2.8 leads to different characterizations of balanced dual frames of a given BF:

Proposition 4.13. Let $\mathcal{F}=\left(f_{k}\right)_{k=1}^{K}$ be a BF for $\mathbb{H}_{d}$ and $\mathcal{G}=\left(g_{k}\right)_{k=1}^{K}$ be a sequence in $\mathbb{H}_{d}$. Then the following are equivalent:

(1) $\mathcal{G}$ is a balanced dual frame of $\mathcal{F}$.

(2) $T_{\mathcal{G}}=S_{\mathcal{F}}^{-1} T_{\mathcal{F}}+R$ where $R \in \mathcal{L}\left(\mathbb{F}^{K}, \mathbb{H}_{d}\right), R T_{\mathcal{F}}^{*}=0$ and $R e=0$.

(3) $\left(g_{k}\right)_{k=1}^{K}=\left(S_{\mathcal{F}}^{-1} f_{k}+r_{k}\right)_{k=1}^{K}$ with $\left(r_{k}\right)_{k=1}^{K}$ in $\mathbb{H}_{d}$ such that $\sum_{k=1}^{K}\left\langle f, f_{k}\right\rangle r_{k}=0$ for each $f \in \mathbb{H}_{d}$ and $\sum_{k=1}^{K} r_{k}=0$.

(4) $T_{\mathcal{G}}=S_{\mathcal{F}}^{-1} T_{\mathcal{F}}+R$, where $R \in \mathcal{L}\left(\mathbb{F}^{K}, \mathbb{H}_{d}\right)$ and $\operatorname{span}\{e\} \oplus \operatorname{im}\left(T_{\mathcal{F}}^{*}\right) \subset \operatorname{ker}(R)$.

(5) $T_{\mathcal{G}}=S_{\mathcal{F}}^{-1} T_{\mathcal{F}}+R$, where $R \in \mathcal{L}\left(\mathbb{F}^{K}, \mathbb{H}_{d}\right)$ and $\operatorname{im}\left(R^{*}\right) \oplus i m\left(T_{\mathcal{F}}^{*}\right) \subset \operatorname{span}\{e\}^{\perp}$

(6) $T_{\mathcal{G}}=S_{\mathcal{F}}^{-1} T_{\mathcal{F}}+W\left(I-T_{\mathcal{F}}^{*} S_{\mathcal{F}}^{-1} T_{\mathcal{F}}\right)$, where $W \in \mathcal{L}\left(\mathbb{F}^{K}, \mathbb{H}_{d}\right)$ and $W e=0$.

(7) $\left(g_{k}\right)_{k=1}^{K}=\left(S_{\mathcal{F}}^{-1} f_{k}+h_{k}+\sum_{l=1}^{K}\left\langle S_{\mathcal{F}}^{-1} f_{k}, f_{l}\right\rangle h_{l}\right)_{k=1}^{K}$ with $\left(h_{k}\right)_{k=1}^{K}$ in $\mathbb{H}_{d}$ such that $\sum_{k=1}^{K} h_{k}=0$.

Corollary 4.14. Let $\mathcal{F}=\left(f_{k}\right)_{k=1}^{K}$ be a BF for $\mathbb{H}_{d}$. Let $\mathcal{G}$ be a balanced dual frame of $\mathcal{F}$ with $T_{\mathcal{G}}=S_{\mathcal{F}}^{-1} T_{\mathcal{F}}+R$, where $R \in \mathcal{L}\left(\mathbb{F}^{K}, \mathbb{H}_{d}\right)$. Then $\operatorname{rank}(R) \leq K-d-1$.

As a consequence of Proposition 4.13 we also have the following uniqueness result:

Corollary 4.15. Let $\mathcal{F}=\left(f_{k}\right)_{k=1}^{K}$ be a BF for $\mathbb{H}_{d}$. Then $K=d+1$ if and only if $\left(S_{\mathcal{F}}^{-1} f_{k}\right)_{k=1}^{K}$ is the unique balanced dual frame of $\left(f_{k}\right)_{k=1}^{K}$.

The above corollary suggests that in the family of BFs, those BFs with $K=d+1$ can be seen as the analogous to the bases in the family of frames.

The existence of distinct types of dual frames of a given Parseval frame is studied in 10. In particular, it is shown that a Parseval frame is itself its unique Parseval dual frame. They also consider for a given Parseval frame its tight dual frames. Here we are interested in balanced tight dual frames of a given balanced Parseval frame.

Theorem 4.16. Let $\mathcal{F}=\left(f_{k}\right)_{k=1}^{K}$ be a BPF for $\mathbb{H}_{d}$. If $K \leq 2 d$ the unique balanced tight dual frame of $\mathcal{F}$ is $\mathcal{F}$. If $K>2 d$ there exist infinite non unitary equivalent balanced tight dual frames of $\mathcal{F}$.

Proof. Let $\mathcal{G}$ be a balanced $\alpha$-tight dual frame of $\mathcal{F}$. Assume $T_{\mathcal{G}}=T_{\mathcal{F}}+R$ with $R \in \mathcal{L}\left(\mathbb{F}^{K}, \mathbb{H}_{d}\right)$ such that $R T_{\mathcal{F}}^{*}=0$ and $R e=0$. Then

$$
\alpha I_{\mathbb{H}_{d}}=I_{\mathbb{H}_{d}}+R R^{*} .
$$

Thus, $\alpha \neq 1$ (indeed $\alpha>1$ ) if and only if $\operatorname{rank}(R)=d$, and $\alpha=1$ if and only if $R=0$.

If $\operatorname{rank}(R)=d$, by Corollary 4.14, $K \geq 2 d+1$. So, if $K \leq 2 d$ the unique balanced tight dual frame of $\mathcal{F}$ is $\mathcal{F}$.

Let $\left\{e_{1}, \ldots, e_{d}\right\}$ be any orthonormal basis of $\mathbb{H}_{d}$. If $K>2 d$, we can consider any set of equal norm orthogonal vectors $\left\{s_{1}, \ldots, s_{d}\right\} \subset\left(\operatorname{span}\{e\} \oplus \operatorname{im}\left(T_{\mathcal{F}}^{*}\right)\right)^{\perp}$. Let $\mathcal{R}=\left(r_{k}\right)_{k=1}^{K}$ with $r_{k}=$ $\sum_{i=1}^{d} \overline{s_{i}(k)} e_{i}$ for $k=1, \ldots, K$. Then

If $f \in \mathbb{H}_{d}$,

$$
T_{\mathcal{R}} e=\sum_{k=1}^{K} r_{k}=\sum_{i=1}^{d} \sum_{k=1}^{K} \overline{s_{i}(k)} e_{i}=0 .
$$

and

$$
T_{\mathcal{R}} T_{\mathcal{F}}^{*} f=\sum_{k=1}^{K}\left\langle f, f_{k}\right\rangle r_{k}=\sum_{i=1}^{d} \sum_{k=1}^{K}\left\langle f, f_{k}\right\rangle \overline{s_{i}(k)} e_{i}=0
$$

$$
T_{\mathcal{R}} T_{\mathcal{R}}^{*} f=\sum_{k=1}^{K}\left\langle f, r_{k}\right\rangle r_{k}=\sum_{i, i^{\prime}=1}^{d}\left\langle f, e_{i}\right\rangle e_{i^{\prime}} \sum_{k=1}^{K} s_{i}(k) \overline{s_{i^{\prime}}(k)}=\rho \sum_{i=1}^{d}\left\langle f, e_{i}\right\rangle e_{i}=\rho f
$$


where $\rho=\left\|s_{i}\right\|^{2}$ for $i=1, \ldots, d$. Thus $\left(f_{k}+r_{k}\right)_{k=1}^{K}$ is a balanced $(\rho+1)$-tight dual frame of $\mathcal{F}$ with Gram matrix $G_{\mathcal{F}}+\rho I \neq G_{\mathcal{F}}$. This shows that if $K>2 d$ there exist infinite non unitary equivalent balanced tight dual frames of $\mathcal{F}$.

\section{The ClOSEST BALANCED FRAME TO A GIVEN FRAME}

A natural question that arises is: Given a frame, is there a balanced frame that is closest to it in some norm and how do we find it? The first step to answer this question is the following theorem, that describes the $\ell^{1}$-norm closest balanced sequence to a given sequence of elements in $\mathbb{H}_{d}$. We consider the $\ell^{1}$-norm of a sequence in $\mathbb{H}_{d}$ given by $\left\|\left(f_{k}\right)_{k=1}^{K}\right\|_{1}:=\sum_{k=1}^{K}\left\|f_{k}\right\|$.

Theorem 5.1. Let $\left(f_{k}\right)_{k=1}^{K}$ be a sequence in $\mathbb{H}_{d}$. Then

$$
\left\|\sum_{k=1}^{K} f_{k}\right\|=\inf \left\{\sum_{k=1}^{K}\left\|f_{k}-g_{k}\right\|:\left(g_{k}\right)_{k=1}^{K} \text { is a balanced sequence in } \mathbb{H}_{d}\right\} \text {, }
$$

and the infimum is attained for the sequences of the form $\left(f_{k}-p_{k} \sum_{l=1}^{K} f_{l}\right)_{k=1}^{K}$, where $0<p_{k}<1$ for each $k=1, \ldots, K$ and $\sum_{k=1}^{K} p_{k}=1$.

Proof. Let $\left(g_{k}\right)_{k=1}^{K}$ be a balanced sequence in $\mathbb{H}_{d}$, and $0<p_{k}<1$ for each $k=1, \ldots, K$ with $\sum_{k=1}^{K} p_{k}=1$. We have,

$$
\sum_{k=1}^{K}\left\|f_{k}-\left(f_{k}-p_{k} \sum_{l=1}^{K} f_{l}\right)\right\|=\left\|\sum_{k=1}^{K} f_{k}\right\|=\left\|\sum_{k=1}^{K} f_{k}-\sum_{k=1}^{K} g_{k}\right\| \leq \sum_{k=1}^{K}\left\|f_{k}-g_{k}\right\| \text {. }
$$

Now suppose that $\left(g_{k}\right)_{k=1}^{K}$ is a balanced sequence in $\mathbb{H}_{d}$ and $\sum_{k=1}^{K}\left\|f_{k}-g_{k}\right\|=\left\|\sum_{k=1}^{K} f_{k}\right\|$. Then $\sum_{k=1}^{K}\left\|f_{k}-g_{k}\right\|=\left\|\sum_{k=1}^{K}\left(f_{k}-g_{k}\right)\right\|$, and this happens if and only if there exist positive real numbers $c_{1}, \ldots, c_{K-1}$ such that $f_{k+1}-g_{k+1}=c_{k}\left(f_{1}-g_{1}\right)$ for each $k=1, \ldots, K-1$. Setting $p_{1}=\frac{1}{1+c_{1}+\ldots+c_{K-1}}$ and $p_{k+1}=\frac{c_{k}}{1+c_{1}+\ldots+c_{K-1}}$ for each $k=1, \ldots, K-1$ we have $\sum_{k=1}^{K} p_{k}=1$, $0<p_{k}<1$ and $g_{k}=f_{k}-p_{k} \sum_{l=1}^{K} f_{l}$ for each $k=1, \ldots, K$.

Now we analyze the problem for the $\ell^{2}$-norm. Given a sequence $\left(f_{k}\right)_{k=1}^{K}$ in $\mathbb{H}_{d}$, the next theorem asserts that $\left(f_{k}-\frac{1}{K} \sum_{l=1}^{K} f_{l}\right)_{k=1}^{K}$ is the balanced sequence in $\mathbb{H}_{d}$ closest to $\left(f_{k}\right)_{k=1}^{K}$ in the $\ell^{2}$-norm, where $\left\|\left(f_{k}\right)_{k=1}^{K}\right\|_{2}:=\left(\sum_{k=1}^{K}\left\|f_{k}\right\|^{2}\right)^{1 / 2}$. In its proof we use the following equality:

$$
\sum_{1 \leq k<k^{\prime} \leq K}\left\|f_{k}-f_{k^{\prime}}\right\|^{2}+\left\|\sum_{l=1}^{K} f_{l}\right\|^{2}=K \sum_{k=1}^{K}\left\|f_{k}\right\|^{2} .
$$

Theorem 5.2. Let $\left(f_{k}\right)_{k=1}^{K}$ be a sequence in $\mathbb{H}_{d}$. Then

$\frac{1}{K}\left\|\sum_{l=1}^{K} f_{l}\right\|^{2}=\inf \left\{\sum_{k=1}^{K}\left\|f_{k}-g_{k}\right\|^{2}:\left(g_{k}\right)_{k=1}^{K}\right.$ is a balanced sequence in $\left.\mathbb{H}_{d}\right\}$, and the infimum is attained for $g_{k}=f_{k}-\frac{1}{K} \sum_{l=1}^{K} f_{l}$ for each $k=1, \ldots, K$.

Proof. Let $\left(g_{k}\right)_{k=1}^{K}$ be a balanced sequence in $\mathbb{H}_{d}$. Using (5.1),

$$
\begin{aligned}
\sum_{k=1}^{K}\left\|f_{k}-\left(f_{k}-\frac{1}{K} \sum_{l=1}^{K} f_{l}\right)\right\|^{2} & =\frac{1}{K}\left\|\sum_{k=1}^{K} f_{k}\right\|^{2} \\
& =\frac{1}{K}\left\|\sum_{k=1}^{K} f_{k}-\sum_{k=1}^{K} g_{k}\right\|^{2} \\
& \leq \frac{1}{K}\left[\left\|\sum_{k=1}^{K}\left(f_{k}-g_{k}\right)\right\|^{2}+\sum_{1 \leq k<k^{\prime} \leq K}\left\|\left(f_{k}-g_{k}\right)-\left(f_{k^{\prime}}-g_{k^{\prime}}\right)\right\|^{2}\right] \\
& =\sum_{k=1}^{K}\left\|f_{k}-g_{k}\right\|^{2} .
\end{aligned}
$$


Now suppose that $\left(g_{k}\right)_{k=1}^{K}$ is a balanced sequence in $\mathbb{H}_{d}$ and $\sum_{k=1}^{K}\left\|f_{k}-g_{k}\right\|^{2}=\frac{1}{K}\left\|\sum_{k=1}^{K} f_{k}\right\|^{2}$. Then $\sum_{k=1}^{K}\left\|f_{k}-g_{k}\right\|^{2}=\frac{1}{K}\left\|\sum_{k=1}^{K}\left(f_{k}-g_{k}\right)\right\|^{2}$. So, by (5.1), $f_{1}-g_{1}=\ldots=f_{K}-g_{K}$. Therefore, $g_{k}=f_{k}-\frac{1}{K} \sum_{l=1}^{K} f_{l}$ for each $k=1, \ldots, K$.

Note that if $\mathcal{F}=\left(f_{k}\right)_{k=1}^{K}$ and $\mathcal{G}=\left(g_{k}\right)_{k=1}^{K}$, then $\sum_{k=1}^{K}\left\|f_{k}-g_{k}\right\|^{2}=\left\|T_{\mathcal{F}}-T_{\mathcal{G}}\right\|_{F}^{2}$, where $\|\cdot\|_{F}$ denotes the Frobenius norm. In order to apply the above theorems to frames we have the following result.

Lemma 5.3. Let $\left(p_{1}, \ldots, p_{K}\right)^{t} \in \mathbb{R}^{K}$, where $0<p_{k}<1$ for each $k=1, \ldots, K$ and $\sum_{k=1}^{K} p_{k}=$ 1. If $\mathcal{F}=\left(f_{k}\right)_{k=1}^{K}$ is a frame for $\mathbb{H}_{d}$, then $\left(f_{k}-p_{k} \sum_{l=1}^{K} f_{l}\right)_{k=1}^{K}$ is a BF for $\mathbb{H}_{d}$ if and only if $\left(p_{1}, \ldots, p_{K}\right)^{t} \notin i m\left(T_{\mathcal{F}}^{*}\right)$.

Proof. The synthesis operator of $\left(f_{k}-p_{k} \sum_{l=1}^{K} f_{l}\right)_{k=1}^{K}$ is $T_{\mathcal{F}}\left(I-e\left(p_{1}, \ldots, p_{K}\right)\right)$. If $\mathcal{F}$ is a frame for $\mathbb{H}_{d}$, by the Sylvester inequality [22], $d-1 \leq \operatorname{rank}\left(T_{\mathcal{F}}\left(I-e\left(p_{1}, \ldots, p_{K}\right)\right)\right) \leq d$, and by the Wedderburn's rank-one reduction formula [22], $\operatorname{rank}\left(T_{\mathcal{F}}-T_{\mathcal{F}} e\left(p_{1}, \ldots, p_{K}\right)\right)=d-1$ if and only if $\left(p_{1}, \ldots, p_{K}\right)^{t} \in \operatorname{im}\left(T_{\mathcal{F}}^{*}\right)$.

Remark 5.4. Let $\mathcal{F}=\left(f_{k}\right)_{k=1}^{K}$ be a frame for $\mathbb{H}_{d}$. Let $\left(p_{1}, \ldots, p_{K}\right)^{t} \in \mathbb{R}^{K}$, where $0<p_{k}<1$ for each $k=1, \ldots, K$ and $\sum_{k=1}^{K} p_{k}=1$. If there exists $f \in \mathbb{H}_{d}$ such that $\left(p_{1}, \ldots, p_{K}\right)^{t}=T_{\mathcal{F}}^{*} f$, then $\left(f_{k}-p_{k} \sum_{l=1}^{K} f_{l}\right)_{k=1}^{K}$ is a BF for $\operatorname{span}\{f\}^{\perp}$. Conversely, if there exists $f \in \mathbb{H}_{d}, f \notin \operatorname{ker}\left(T_{\mathcal{F}}^{*}\right)$, such that $\left(f_{k}-p_{k} \sum_{l=1}^{K} f_{l}\right)_{k=1}^{K}$ is a BF for $\operatorname{span}\{f\}^{\perp}$, then $\left(p_{1}, \ldots, p_{K}\right)^{t}=\gamma T_{\mathcal{F}}^{*} f$ for some $\gamma \in \mathbb{F}, \gamma \neq 0$.

We can now give the answer to the question we posed at the beginning of this section. The following theorem gives necessary and sufficient conditions for the closest balanced frame to a given frame to exist, and in this case gives its expression.

Theorem 5.5. Let $\mathcal{F}=\left(f_{k}\right)_{k=1}^{K}$ be a frame for $\mathbb{H}_{d}$. Then

(1) There exist $\ell^{1}$-norm closest to $\mathcal{F}$ balanced frames for $\mathbb{H}_{d}$ if and only if $\mathcal{F}$ is not a basis, and in this case they are the frames $\left(f_{k}-p_{k} \sum_{l=1}^{K} f_{l}\right)_{k=1}^{K}$ where $0<p_{k}<1, \sum_{k=1}^{K} p_{k}=1$ and $\left(p_{1}, \ldots, p_{K}\right)^{t} \notin i m\left(T_{\mathcal{F}}^{*}\right)$.

(2) There exist an $\ell^{2}$-norm closest to $\mathcal{F}$ balanced frame for $\mathbb{H}_{d}$ if and only if $e \notin i m\left(T_{\mathcal{F}}^{*}\right)$, and in this case it is the frame $\left(f_{k}-\frac{1}{K} \sum_{l=1}^{K} f_{l}\right)_{k=1}^{K}$.

Proof. If $\mathcal{F}$ is a basis, clearly there does not exist an $\ell^{1}$-norm $\left(\ell^{2}\right.$-norm) closest to $\mathcal{F}$ balanced sequence which is a frame for $\mathbb{H}_{d}$, since there does not exist balanced frames for $\mathbb{H}_{d}$ with $K=d$ elements. Thus we suppose that $\mathcal{F}$ is not a basis.

(1) Since the set $\left\{\left(p_{1}, \ldots, p_{K}\right)^{t} \in \mathbb{R}^{K}: 0<p_{k}<1\right.$ and $\left.\sum_{k=1}^{K} p_{k}=1\right\} \cap \operatorname{im}\left(T_{\mathcal{F}}^{*}\right)^{c}$ has an infinite number of points, the conclusion follows from Theorem 5.1 and Lemma 5.3

(2) By Theorem 5.2 and Lemma [5.3, if $e \notin \operatorname{im}\left(T_{\mathcal{F}}^{*}\right)$ then $\left(f_{k}-\frac{1}{K} \sum_{l=1}^{K} f_{l}\right)_{k=1}^{K}$ is the $\ell^{2}$-norm closest to $\mathcal{F}$ balanced frame for $\mathbb{H}_{d}$.

Now suppose that $e \in \operatorname{im}\left(T_{\mathcal{F}}^{*}\right)$. Let $\mathcal{G}=\left(g_{k}\right)_{k=1}^{K}$ be any BF for $\mathbb{H}_{d}$. We are going to prove that we can always find another $\mathrm{BF}$ for $\mathbb{H}_{d}$ closer to $\mathcal{F}$ than $\mathcal{G}$ in the $\ell^{2}$-norm.

Suppose without loss of generality that $\mathcal{F}_{2}=\left(f_{k}\right)_{k=2}^{K}$ still generates $\mathbb{H}_{d}$, that is, $\mathcal{F}_{2}$ is a frame for $\mathbb{H}_{d}$. So, $T_{\mathcal{F}_{2}}^{*}$ is injective.

For $\epsilon \neq 0$ let $\mathcal{F}_{\epsilon}=\left(f_{\epsilon, k}\right)_{k=1}^{K}$ where $f_{\epsilon, 1}=\epsilon f_{1}, f_{\epsilon, 2}=f_{2}, \ldots, f_{\epsilon, K}=f_{K}$.

Since $e \in \operatorname{im}\left(T_{\mathcal{F}}^{*}\right)$, none of the elements of $\mathcal{F}$ is the null vector. Also, by Lemma 5.3. $\widetilde{\mathcal{F}}=$ $\left(f_{k}-\frac{1}{K} \sum_{l=1}^{K} f_{l}\right)_{k=1}^{K}$ is not a frame for $\mathbb{H}_{d}$. So $\mathcal{G} \neq \widetilde{\mathcal{F}}$ and, by Theorem 5.2 ,

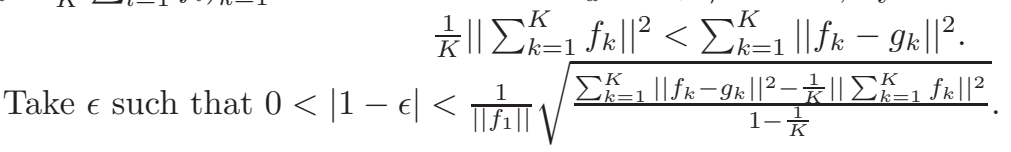


Let $f \in \mathbb{H}_{d}$ such that $T_{\mathcal{F}}^{*} f=e$. Then $T_{\mathcal{F}_{2}}^{*} f=(1, \ldots, 1)^{t}$. If there would exist $g \in \mathbb{H}_{d}$ such that $T_{\mathcal{F}_{\epsilon}}^{*} g=e$, then $T_{\mathcal{F}_{2}}^{*} g=(1, \ldots, 1)^{t}$. Since $T_{\mathcal{F}_{2}}^{*}$ is injective $g=f$. So, $\left\langle f, f_{1}\right\rangle=\left\langle f, \epsilon f_{1}\right\rangle=1$. Thus $\epsilon=$ 1 which is absurd. This shows that $e \notin \operatorname{im}\left(T_{\mathcal{F}_{\epsilon}}^{*}\right)$. Hence, by Lemma $5.3, \widetilde{\mathcal{F}}_{\epsilon}=\left(f_{\epsilon, k}-\frac{1}{K} \sum_{l=1}^{K} f_{\epsilon, l}\right)_{k=1}^{K}$ is a $\mathrm{BF}$ for $\mathbb{H}_{d}$.

We have,

$$
\begin{aligned}
\sum_{k=1}^{K} \| f_{k}-\left(f_{\epsilon, k}-\right. & \left.\frac{1}{K} \sum_{l=1}^{K} f_{\epsilon, l}\right)\left\|^{2}=\right\|(1-\epsilon)\left(1-\frac{1}{K}\right) f_{1}+\frac{1}{K} \sum_{l=1}^{K} f_{l}\left\|^{2}+\sum_{k=2}^{K}\right\| \frac{1}{K}(\epsilon-1) f_{1}+\frac{1}{K} \sum_{l=1}^{K} f_{l} \|^{2} \\
= & |1-\epsilon|^{2}\left(1-\frac{1}{K}\right)^{2}\left\|f_{1}\right\|^{2}+2(1-\epsilon) \frac{K-1}{K^{2}} \operatorname{Re}\left(\left\langle f_{1}, \sum_{l=1}^{K} f_{l}\right\rangle\right)+\frac{1}{K^{2}}\left\|\sum_{l=1}^{K} f_{l}\right\|^{2} \\
& +\sum_{k=2}^{K}\left[\frac{1}{K^{2}}|\epsilon-1|^{2}\left\|f_{1}\right\|^{2}+2 \frac{1}{K^{2}}(\epsilon-1) \operatorname{Re}\left(\left\langle f_{1}, \sum_{l=1}^{K} f_{l}\right\rangle\right)+\frac{1}{K^{2}}\left\|\sum_{l=1}^{K} f_{l}\right\|^{2}\right] \\
= & |1-\epsilon|^{2}\left(1-\frac{1}{K}\right)\left\|f_{1}\right\|^{2}+\frac{1}{K}\left\|\sum_{l=1}^{K} f_{l}\right\|^{2}<\sum_{k=1}^{K}\left\|f_{k}-g_{k}\right\|^{2} .
\end{aligned}
$$

Hence $\widetilde{\mathcal{F}}_{\epsilon}$ is a BF for $\mathbb{H}_{d}$ closer to $\mathcal{F}$ in the $\ell^{2}$-norm than $\mathcal{G}$.

\section{A NEW CONCEPt OF COMPLEMENT FOR BALANCED FRAMES}

Let $\mathcal{F}$ be a BENPF for $\mathbb{H}_{d}$ and $\mathcal{G}$ be any of its complements. Then $\mathcal{G}$ is an ENPF for $\mathbb{H}_{d}$. Since $G_{\mathcal{G}} e=e$, by Proposition $2.8 \mathcal{G}$ is not balanced. Morevover, since $e \in \operatorname{im}\left(T_{\mathcal{G}}^{*}\right)$, Theorem 5.5 tells us that although $\mathcal{G}$ has closest balanced frames in the $\ell^{1}$-norm, it has not a closest balanced frame in the $\ell^{2}$-norm.

In order to have complementary frames in the same class, we define an alternative concept of complements for BPFs. To this end, we first state the following proposition whose proof is straightforward.

Proposition 6.1. Let $\mathcal{F}=\left(f_{k}\right)_{k=1}^{K}$ be a BPF for $\mathbb{H}_{d}$. Then $I-G_{\mathcal{F}}-\frac{1}{K} e e^{t}$ is the orthogonal projection onto $\left(i m\left(G_{\mathcal{F}}\right) \oplus \operatorname{span}\{e\}\right)^{\perp}$.

Note that $\operatorname{rank}\left(I-G_{\mathcal{F}}-\frac{1}{K} e e^{t}\right)=K-d-1$ and $\left(I-G_{\mathcal{F}}-\frac{1}{K} e e^{t}\right) e=0$. Based on Proposition 6.1 and Theorem 4.10 we introduce the following definition:

Definition 6.2. Two PFs $\mathcal{F}$ and $\mathcal{G}$ are $B$-complements of each other if the sum of their Gramians is $I-\frac{1}{K} e e^{t}$.

In view of Proposition 6.1 the B-complement of a BPF of $K$ vectors for a space of dimension $d$ is a BPF of $K$ vectors for a space of dimension $K-d-1$. For future references we state the following lemma that follows immediately from the definitions of simplex frame and of B-complement:

Lemma 6.3. $\mathcal{F}$ is a simplex frame with $K$ elements if and only if its $B$-complement is the frame for the zero vector space given by the zero vector repeated $K$ times.

Note that the sum of the Gram matrices of two complementary PFs is $I$, which is the Gram matrix corresponding to an orthonormal basis. By Proposition 2.5. an orthonormal basis can be seen as a "limit case" of a PF: it is a UNPF or a PF with $K=d$. In the case of two Bcomplementary BPFs, the sum of their Gram matrices is $I-\frac{1}{K} e e^{t}$, which is the Gram matrix corresponding to simplex frames. We can think that in the family of BFs, simplex frames are the analogous to othonormal basis in the family of frames. This follows from Theorem 6.4 below, which 
shows that a simplex frame can be seen as a "limit case" of BPF: it is a BENPF which elements have norm equal to $\sqrt{\frac{d}{d+1}}$, or a BPF with $K=d+1$.

Theorem 6.4. Let $\mathcal{F}=\left(f_{k}\right)_{k=1}^{K}$ be a sequence in $\mathbb{H}_{d}$. The following assertions are equivalent:

(1) $\mathcal{F}$ is a simplex frame for $\mathbb{H}_{d}$.

(2) $\mathcal{F}$ is a BPF for $\mathbb{H}_{d}$ and $K=d+1$.

(3) $\mathcal{F}$ is a BPF for $\mathbb{H}_{d}$ and $\left\|f_{k}\right\|^{2}=\frac{d}{d+1}$ for each $k=1, \ldots, K$.

(4) $\mathcal{F}$ is an isogonal $P F$ for $\mathbb{H}_{d}$ with $K>d$ and $\left\|f_{k}\right\|^{2} \neq\left\langle f_{k}, f_{l}\right\rangle$ for each $k=1, \ldots, K, k \neq l$.

(5) $\mathcal{F}$ is a BPF with $\operatorname{ker}\left(T_{\mathcal{F}}\right)=\operatorname{span}\{e\}$.

Proof. If $\mathcal{F}$ is a simplex frame for $\mathbb{H}_{d}$ then, by Proposition 2.5 and Corollary 4.5 $\mathcal{F}$ is an isogonal BPF for $\mathbb{H}_{d}$ and $K=d+1$. We also have $\operatorname{diag}\left(G_{\mathcal{F}}\right)=1-\frac{1}{K}=\frac{d}{d+1}$ and $\operatorname{ker}\left(T_{\mathcal{F}}\right)=\operatorname{ker}\left(G_{\mathcal{F}}\right)=$ $\operatorname{span}\{e\}$. So (1) implies the rest of the assertions.

$(2) \Rightarrow(1)$. Suppose that $\mathcal{F}$ is a BPF with $K=d+1$. Let $\mathcal{G}$ be a B-complement of $\mathcal{F}$. Then $\operatorname{rank}\left(G_{\mathcal{G}}\right)=K-d-1=0$. So, by Lemma 6.3, $\mathcal{F}$ is a simplex frame.

$(3) \Rightarrow(2)$. Suppose that $\mathcal{F}$ is BPF for $\mathbb{H}_{d}$ and $\left\|f_{k}\right\|^{2}=\frac{d}{d+1}$ for each $k=1, \ldots, K$. Then $\left(\sqrt{\frac{d+1}{d}} f_{k}\right)_{k=1}^{K}$ is a $\frac{d+1}{d}$-BUNTF. From Proposition 2.5, $\frac{d+1}{d}=\frac{K}{d}$. So, $K=d+1$.

(4) $\Rightarrow(1)$. By hypotheses, $G_{\mathcal{F}}^{2}=G_{\mathcal{F}}$ and there exists $a, c \in \mathbb{R}, a \neq c$, such that $G_{\mathcal{F}}=$ $(c-a) I+a e e^{t} . G_{\mathcal{F}}$ is a circulant matrix, so its eigenvalues are $c+a(K-1)$ and $c-a$ with multiplicity $K-1$ [22]. Since $\operatorname{rank}\left(G_{\mathcal{F}}\right)=d, K>d$ and $c-a \neq 0$, we have $a=-\frac{c}{K-1}$ and $K-1=d$. Thus $G_{\mathcal{F}}=\frac{c}{K-1}\left(K I-e e^{t}\right)$. Since $G_{\mathcal{F}}^{2}=G_{\mathcal{F}}, c=\frac{K-1}{K}$. Therefore, $G_{\mathcal{F}}=I-\frac{1}{K} e e^{t}$ and $\mathcal{F}$ is a simplex frame.

$(5) \Rightarrow(1)$. By the hypotheses, $G_{\mathcal{F}}$ is an orthogonal projection matrix and $\operatorname{im}\left(G_{\mathcal{F}}\right)=\operatorname{span}\{e\}^{\perp}$, so $G_{\mathcal{F}}=I-\frac{1}{K} e e^{t}$ and $\mathcal{F}$ is a simplex frame.

Some of the points of the previous theorem can be seen as variations of statements that appear in [34. By Corollary 4.15 and Theorem 6.4, the canonical dual, which in this case it is itself, is the unique balanced dual of a simplex frame. Moreover, by Theorem 6.4 and Corollary 4.3,

Corollary 6.5. $\mathcal{F}$ is a $B F$ for $\mathbb{H}_{d}$ with $K=d+1$ if and only if $S_{\mathcal{F}}^{-1 / 2} \mathcal{F}$ is a simplex frame for $\mathbb{H}_{d}$.

In what follows we consider properties of B-complementary BPFs that are analogous to properties of complementary PFs that can be found in 34 .

Let $\mathcal{F}$ be a BPF for $\mathbb{H}_{d}$. The B-complements of $\mathcal{F}$ are unitary equivalent. Let $\mathcal{G}$ be a Bcomplement of $\mathcal{F}$. Then $\mathcal{F}$ is equal-norm (or isogonal or real) if and only if $\mathcal{G}$ is. $\mathcal{F}$ and $\mathcal{G}$ can not be unitarily equivalent.

We note that if $\mathcal{F}$ is a BPF for $\mathbb{F}^{d}$ with $K$ elements and $\left(\frac{1}{\sqrt{K}} e, v_{1}, \ldots, v_{K-d-1}\right)$ is an orthonormal basis for $\operatorname{ker}\left(T_{\mathcal{F}}\right)$, then the columns of the matrix which rows are $v_{k}^{*}, k=1, \ldots, K-d-1$, constitutes a B-complement BPF of $\mathcal{F}$.

We now introduce B-complementary BFs:

Definition 6.6. Two BFs $\mathcal{F}$ and $\mathcal{G}$ are B-complements if the PFs $S_{\mathcal{F}}^{-1 / 2} \mathcal{F}$ and $S_{\mathcal{G}}^{-1 / 2} \mathcal{G}$ are Bcomplements.

Analogous to [34, Proposition 5.1] we have:

Proposition 6.7. Let $\mathcal{F}=\left(f_{k}\right)_{k=1}^{K}$ and $\mathcal{G}=\left(g_{k}\right)_{k=1}^{K}$ be BFs for $\mathbb{H}_{d_{1}}$ and $\mathbb{H}_{d_{2}}$, respectively. Then the following are equivalent:

(1) $\mathcal{F}$ and $\mathcal{G}$ are B-complements. 
(2) $i m\left(G_{\mathcal{F}}\right) \oplus i m\left(G_{\mathcal{G}}\right)=\operatorname{span}\{e\}^{\perp}$.

(3) $\operatorname{dim}\left(\mathbb{H}_{d_{1}}\right)+\operatorname{dim}\left(\mathbb{H}_{d_{1}}\right)=K-1$ and $T_{\mathcal{G}} T_{\mathcal{F}}^{*}=0$.

(4) The inner sum $\mathcal{F} \oplus \mathcal{G}=\left(f_{k}, g_{k}\right)_{k=1}^{K}$ is a BF for $\mathbb{H}_{d_{1}} \oplus \mathbb{H}_{d_{2}}$ with $K=d_{1}+d_{2}+1$ and $T_{\mathcal{G}} T_{\mathcal{F}}^{*}=0$.

(5) $T_{\mathcal{G}}=T_{\mathcal{G}}\left(I-\frac{1}{K} e e^{t}-T_{\mathcal{F}}^{*} S_{\mathcal{F}}^{-1} T_{\mathcal{F}}\right)$.

Remark 6.8. In case that $\mathcal{F}=\left(f_{k}\right)_{k=1}^{K}$ and $\mathcal{G}=\left(g_{k}\right)_{k=1}^{K}$ are BPFs, (4) of the previous proposition becomes: $\mathcal{F} \oplus \mathcal{G}=\left(f_{k}, g_{k}\right)_{k=1}^{K}$ is a simplex frame for $\mathbb{H}_{d_{1}} \oplus \mathbb{H}_{d_{2}}$.

This concept can be applied to construct BUNTFs of $K$ vectors for a space of dimension $K-d-1$ from BUNTFs of $K$ vectors for a space of dimension $d$.

\section{EXAMPles of BALANCED UNit NORM Tight FRAMES}

The aim of this section is to present various examples of BUNTFs and some of their properties. Sometimes we identify a frame $\mathcal{F}$ for $\mathbb{F}^{d}$ of $K$ elements with the matrix that represents $T_{\mathcal{F}}$ in the standard bases of $\mathbb{F}^{d}$ and $\mathbb{F}^{K}$.

7.1. The case $\mathbb{F}=\mathbb{R}$ and $d=2$. As a consequence of Proposition 3.5 and [20, Lemma 1] we obtain:

Theorem 7.1. The following are equivalent:

(1) $\left(\left(\cos \theta_{k}, \sin \theta_{k}\right)^{t}\right)_{k=1}^{K}$ is a BUNTF for $\mathbb{R}^{2}$.

(2) $\sum_{k=1}^{K} e^{i \theta_{k}}=\sum_{k=1}^{K} e^{2 i \theta_{k}}=0$.

(3) $\sum_{k=1}^{K} e^{i \theta_{k}}=\sum_{1 \leq k_{1}<k_{2} \leq K} e^{2 i \theta_{k_{1}}} e^{2 i \theta_{k_{2}}}=0$.

By Theorem 7.1, the set of vectors coming from the $K$ th roots of unity are BUNTFs for $\mathbb{R}^{2}$ :

Corollary 7.2. If $K \geq 3$ and $\left(e^{i \theta_{k}}\right)_{k=1}^{K}$ are the $K$ th roots of unity, $\left(\left(\cos \theta_{k}, \sin \theta_{k}\right)^{t}\right)_{k=1}^{K}$ is a BUNTF for $\mathbb{R}^{2}$.

In [20, Theorem $\mathrm{A}$ ] the types of spherical $t$-designs in $\mathbb{R}^{2}$ are described. From this result and Proposition 3.5 we have:

Theorem 7.3. For $K=3,4,5$, there is one equivalence class of BUNTF for $\mathbb{R}^{2}$ with $K$ elements. For $K \geq 6$, there are infinite equivalence classes of BUNTF for $\mathbb{R}^{2}$ with $K$ elements.

We have that for $K=3,4,5$ the class corresponding to the frame coming from the $K$ th roots of unity is the unique equivalence class of BUNTFs for $\mathbb{R}^{2}$ with $K$ elements. We can see that for $K \geq 6$ there are infinite equivalence classes as follows. Note first that, by Corollary 7.2, we always have the class corresponding to the $K$ th roots of unity. Now write $K=3 n+s$ where $s \in \mathbb{N}_{0}, 0 \leq s<3$. Then, if $s=0$ there are the classes corresponding to the union of $n$ rotations of the third roots of unity, and there are infinitely many of such classes. If $s=1$, then $K=3 n+1=3(n-1)+4$. So we have the classes corresponding to the union of the 4 th roots of unity and $n-1$ rotations of the third roots of unity. If $s=2$, then $K=3(n-2)+8$ and similar arguments can be used. Writing e.g. $K=4 m+r$ where $r \in \mathbb{N}_{0}, 0 \leq r<4$ and $m \in \mathbb{N}$, or using other decompositions of $K$, we can see that there exist more equivalence classes of BUNTFs.

In what follows we consider several examples of tight frames, some of them well-known, indicating in which cases they turn out to be balanced. 
7.2. Balanced harmonic frames. Let $F$ be the unitary matrix of order $K$ which entries are $F(k, l)=\frac{1}{\sqrt{K}} e^{\frac{2 \pi i(k-1)(l-1)}{K}}$, called Fourier matrix. The ENPFs $T$ consisting of a $d \times K$ submatrix of $F$ are a particular case of the so called harmonic frames. To obtain real ENPFs we must select real rows and complex conjugate pairs of rows from the Fourier matrix $F$. If $T$ does not contain the first row of $F$, then $T$ is also balanced. More general, unlifted harmonic frames are BENPFs and B-complements of unlifted harmonic frames are unlifted harmonic frames. See [34, Chapter 11] for a detailed treatment of harmonic frames.

7.3. BENTFs from Hadamard matrices. A Hadamard matrix $H$ has orthogonal rows and entries \pm 1 [21]. The smallest examples of Hadamard matrices are:

$$
\text { (1), }\left(\begin{array}{cc}
1 & 1 \\
1 & -1
\end{array}\right),\left(\begin{array}{cccc}
1 & 1 & 1 & 1 \\
1 & -1 & 1 & -1 \\
1 & 1 & -1 & -1 \\
1 & -1 & -1 & 1
\end{array}\right) \text {. }
$$

A way for contructing Hadamard matrices is the following: if $H$ is a Hadamard matrix, $\left(\begin{array}{cc}H & H \\ H & -H\end{array}\right)$ is a Hadamard matrix. Hadamard matrices obtained in this manner are known as SylvesterHadamard matrices. If $H$ has order $K$ and we select a submatrix $T$ of order $d \times K$ from $H$, we can get a BENTF.

7.4. Crosses and eutactic stars. The set $\left( \pm u_{1}, \ldots, \pm u_{K}\right)$, where $\left(u_{1}, \ldots, u_{K}\right)$ is an orthonormal basis for $\mathbb{H}_{d}$, is a BUNTF for $\mathbb{H}_{d}$. By Proposition 4.1 the set $\left( \pm \pi_{\mathcal{W}} u_{1}, \ldots, \pm \pi_{\mathcal{W}} u_{K}\right)$, where $\mathcal{W}$ is a subspace of $\mathbb{H}_{d}$, is a BTF for $\mathcal{W}$. If $\mathbb{H}_{d}=\mathbb{R}^{d},\left( \pm u_{1}, \ldots, \pm u_{K}\right)$ is known as a cross and $\left( \pm \pi_{\mathcal{W}} u_{1}, \ldots, \pm \pi_{\mathcal{W}} u_{K}\right)$ is known as an eutactic star (see [15]).

7.5. Partition frames. Let $\eta=\left(\eta_{1}, \ldots, \eta_{n}\right) \in \mathbb{Z}^{n}$ be a partition of $K$, i.e., $K=\eta_{1}+\ldots+\eta_{n}$ and $1 \leq \eta_{1} \leq \ldots \leq \eta_{n}$. The $\eta$-partition frame for $\mathbb{R}^{d}$ with $d=K-n$, is the complement of the $\mathrm{PF}$

$$
\left[\frac{e_{1}}{\sqrt{\eta_{1}}} \ldots \frac{e_{1}}{\sqrt{\eta_{1}}} \ldots \frac{e_{n}}{\sqrt{\eta_{n}}} \ldots \frac{e_{n}}{\sqrt{\eta_{n}}}\right]
$$

of $K$ vectors for $\mathbb{R}^{n}$. Here each $\frac{e_{j}}{\sqrt{\eta_{j}}}$ is repeated $\eta_{j}$ times. An $\eta$-partition frame for $\mathbb{R}^{d}$ has Gram matrix

$$
G=\left(\begin{array}{ccc}
B_{1} & & \\
& \ddots & \\
& & B_{n}
\end{array}\right)
$$

where $B_{j}$ is the $\eta_{j} \times \eta_{j}$ orthogonal projection matrix with $\frac{\eta_{j}-1}{\eta_{j}}$ as diagonal elements and $\frac{-1}{\eta_{j}}$ as non diagonal elements. See 34] for more details of partition frames.

An $\eta$-partition frame is balanced and Parseval. If $n \mid K$ and $\eta_{1}=\ldots=\eta_{n}$ we obtain an equal norm frame.

A $B$-complement $\mathcal{G}$ of an $\left(\eta_{1}, \ldots, \eta_{n}\right)$-partition frame $\mathcal{F}$ of $K$ elements for $\mathbb{R}^{d}$ has Gram matrix $G_{\mathcal{G}}=\left(C_{i, j}\right)$ where $C_{i, j}$ is an $\eta_{i} \times \eta_{j}$ matrix such that the entries of $C_{i, j}$ are $\frac{K-\eta_{i}}{\eta_{i} K}$ if $i=j$ and $-\frac{1}{K}$ if $i \neq j$. For example, if $n=1, \mathcal{F}$ is a simplex frame and $\mathcal{G}$ is the zero vector repeated $K$ times. If $n=2, \mathcal{G}$ is the BPF of $K$ elements for $\mathbb{R}^{1}$ consisting of $-\sqrt{\frac{\eta_{2}}{\eta_{1} K}}$ and $\sqrt{\frac{\eta_{1}}{\eta_{2} K}}$ (or $\sqrt{\frac{\eta_{2}}{\eta_{1} K}}$ and $-\sqrt{\frac{\eta_{1}}{\eta_{2} K}}$ ) repeated $\eta_{1}$ and $\eta_{2}$ times, respectively.

7.6. BUNTFs from spherical designs. We recall that any spherical $(t+1)$-design is a spherical $t$-design and a real spherical 2-design is a BUNTF. In [3, several examples of spherical $t$-design are presented. They include regular $K$-gons on $\mathbb{S}^{1} \subset \mathbb{R}^{2}$, platonic solids in $\mathbb{R}^{3}$, regular potytopes and roots systems in $\mathbb{R}^{d}$, and the set of minimal vectors of the Leech lattice in $\mathbb{R}^{24}$. 


\section{Construction methods For BALANCED Unit NORM TIGHT FRAMES}

In this section we present explicit and painless constructions of an infinite variety of BUNTFs.

We begin by showing under which conditions some well-known methods for constructing frames lead to the obtention of BUNTFs. For properties of these methods see [34, Chapter 5].

We have the inner product in the orthogonal direct sum $\mathbb{H}_{d_{1}} \oplus \mathbb{H}_{d_{2}}$ given by $\left\langle\left(f_{1}, g_{1}\right),\left(f_{2}, g_{2}\right)\right\rangle:=$ $\left\langle f_{1}, f_{2}\right\rangle+\left\langle g_{1}, g_{2}\right\rangle$ for each $\left(f_{1}, g_{1}\right),\left(f_{2}, g_{2}\right) \in \mathbb{H}_{d_{1}} \oplus \mathbb{H}_{d_{2}}$. The following results give ways to obtain a BUNTF combining two or more BUNTFs.

First we obtain BUNTFs as a disjoint union of BUNTFs:

Proposition 8.1. Let $\mathcal{F}=\left(f_{k}\right)_{k=1}^{K}$ be a sequence in $\mathbb{H}_{d_{1}}$ and $\mathcal{G}=\left(g_{l}\right)_{l=1}^{L}$ be a sequence in $\mathbb{H}_{d_{2}}$. Then the disjoint union $\mathcal{F} \cup \mathcal{G}:=\left(\left(f_{k}, 0\right)_{k=1}^{K},\left(0, g_{l}\right)_{l=1}^{L}\right)$ is a BUNTF for $\mathbb{H}_{d_{1}} \oplus \mathbb{H}_{d_{2}}$ if and only $\mathcal{F}$ is a BUNTF for $\mathbb{H}_{d_{1}}, \mathcal{G}$ is a BUNTF for $\mathbb{H}_{d_{2}}$ and $\frac{K}{d_{1}}=\frac{L}{d_{2}}$.

Proof. Noting that $T_{\mathcal{F} \cup \dot{\mathcal{G}}}=T_{\mathcal{F}} \oplus T_{\mathcal{G}}$ and $S_{\mathcal{F} \dot{\mathcal{G}}}=S_{\mathcal{F}} \oplus S_{\mathcal{G}}, \mathcal{F} \cup \mathcal{G}$ is a BUNTF for $\mathbb{H}_{d_{1}} \oplus \mathbb{H}_{d_{2}}$ if and only $\mathcal{F}$ is a BUNTF for $\mathbb{H}_{d_{1}}, \mathcal{G}$ is a BUNTF for $\mathbb{H}_{d_{2}}$, and $\frac{K+L}{d_{1}+d_{2}}=\frac{K}{d_{1}}=\frac{L}{d_{2}}$. This last condition is equivalent to $\frac{K}{d_{1}}=\frac{L}{d_{2}}$.

Note that in view of Theorem 4.12, each BUNTF is the disjoint union of BUNTFs for orthogonal subspaces, given by the vertices of each connected component of the frame graph. This decomposition is unique.

Now we construct BUNTFs as the inner direct sum of BUNTFs:

Proposition 8.2. Let $\alpha, \beta \in \mathbb{F} \backslash\{0\}$. Let $\mathcal{F}=\left(f_{k}\right)_{k=1}^{K}$ be a sequence in $\mathbb{H}_{d_{1}}$ and $\mathcal{G}=\left(g_{k}\right)_{k=1}^{K}$ be a sequence in $\mathbb{H}_{d_{2}}$ be UN. Then the inner direct sum $\alpha \mathcal{F} \oplus \beta \mathcal{G}:=\left(\left(\alpha f_{k}, \beta g_{k}\right)\right)_{k=1}^{K}$ is a BUNTF for $\mathbb{H}_{d_{1}} \oplus \mathbb{H}_{d_{2}}$ if and only if $\mathcal{F}$ is a BUNTF for $\mathbb{H}_{d_{1}}, \mathcal{G}$ is a BUNTF for $\mathbb{H}_{d_{2}}, T_{\mathcal{F}} T_{\mathcal{G}}^{*}=0,|\alpha|^{2}=\frac{d_{1}}{d_{1}+d_{2}}$ and $|\beta|^{2}=\frac{d_{2}}{d_{1}+d_{2}}$.

Proof. We have $T_{\alpha \mathcal{F} \oplus \beta \mathcal{G}}(c)=\left(\alpha T_{\mathcal{F}}(c), \beta T_{\mathcal{G}}(c)\right)$ for all $c \in F^{K}$ and

$$
S_{\alpha \mathcal{F} \oplus \beta \mathcal{G}}(f, g)=\left(|\alpha|^{2} S_{\mathcal{F}}(f)+\alpha \bar{\beta} T_{\mathcal{F}} T_{\mathcal{G}}^{*}(g), \bar{\alpha} \beta T_{\mathcal{G}} T_{\mathcal{F}}^{*}(f)+|\beta|^{2} S_{\mathcal{G}}(g)\right)
$$

for all $f \in \mathbb{H}_{d_{1}}, g \in \mathbb{H}_{d_{2}}$. Therefore $\alpha \mathcal{F} \oplus \beta \mathcal{G}$ is a BUNTF if and only if $\mathcal{F}$ is a BUNTF for $\mathbb{H}_{d_{1}}, \mathcal{G}$ is a BUNTF for $\mathbb{H}_{d_{2}}, T_{\mathcal{F}} T_{\mathcal{G}}^{*}=0$ and $|\alpha|^{2} \frac{K}{d_{1}}=|\beta|^{2} \frac{K}{d_{2}}=\frac{K}{d_{1}+d_{2}}$.

See [34, Lemma 5.1] for equivalent conditions to $T_{\mathcal{F}} T_{\mathcal{G}}^{*}=0$. In particular, this condition implies that $K \geq d_{1}+d_{2}$.

Another way to construct BUNTFs is to take the sum of BUNTFs in the following sense:

Proposition 8.3. Let $\alpha, \beta \in \mathbb{F} \backslash\{0\}$. Let $\mathcal{F}=\left(f_{k}\right)_{k=1}^{K}$ be a UN sequence in $\mathbb{H}_{d_{1}}$ and $\mathcal{G}=\left(g_{l}\right)_{l=1}^{L}$ be a UN sequence in $\mathbb{H}_{d_{2}}$. Then the sum $\alpha \mathcal{F} \hat{+} \beta \mathcal{G}:=\left(\left(\alpha f_{k}, \beta g_{l}\right)\right)_{k, l=1}^{K, L}$ is a BUNTF for $\mathbb{H}_{d_{1}} \oplus \mathbb{H}_{d_{2}}$ if and only $\mathcal{F}$ is a BUNTF for $\mathbb{H}_{d_{1}}, \mathcal{G}$ is a BUNTF for $\mathbb{H}_{d_{2}},|\alpha|^{2}=\frac{d_{1}}{d_{1}+d_{2}}$ and $|\beta|^{2}=\frac{d_{2}}{d_{1}+d_{2}}$.

Proof. For each $l=1, \ldots, L$, set $\mathcal{H}_{l}:=\left(h_{l, k}\right)_{k=1}^{K}$ where $h_{l, k}=g_{l}$ for each $k=1, \ldots, K$. Let $E: \mathbb{F}^{L} \rightarrow \mathbb{F}^{K}$ given by $E(c)=\left(\sum_{l=1}^{L} c_{l}, \ldots, \sum_{l=1}^{L} c_{l}\right)$. The synthesis operator is given by

$$
T_{\alpha \mathcal{F} \widehat{\gamma} \beta \mathcal{G}}(d)=\sum_{l \in K}\left(\alpha T_{\mathcal{F}}(d(l)), \beta T_{\mathcal{H}_{l}}(d(l))\right),
$$

where $d \in\left(\mathbb{F}^{K}\right)^{L}$, and the frame operator is given by

$$
S_{\alpha \mathcal{F} \uparrow \beta \mathcal{G}}(f, g)=\left(L|\alpha|^{2} S_{\mathcal{F}}(f)+\alpha \bar{\beta} T_{\mathcal{F}} E T_{\mathcal{G}}^{*}(g), \bar{\alpha} \beta T_{\mathcal{G}} E^{*} T_{\mathcal{F}}^{*}(f)+K|\beta|^{2} S_{\mathcal{G}}(g)\right),
$$

where $f \in \mathbb{H}_{d_{1}}$ and $g \in \mathbb{H}_{d_{2}}$, respectively. It results that $\alpha \mathcal{F} \hat{+} \beta \mathcal{G}$ is a BUNTF for $\mathbb{H}_{d_{1}} \oplus \mathbb{H}_{d_{2}}$ if and only if $\mathcal{F}$ is a BUNTF for $\mathbb{H}_{d_{1}}, \mathcal{G}$ is a BUNTF for $\mathbb{H}_{d_{2}}, L|\alpha|^{2} \frac{K}{d_{1}}=K|\beta|^{2} \frac{L}{d_{2}}=\frac{K L}{d_{1}+d_{2}}$, or equivalently, $|\alpha|^{2}=\frac{d_{1}}{d_{1}+d_{2}}$ and $|\beta|^{2}=\frac{d_{2}}{d_{1}+d_{2}}$. 
In the tensor product $\mathbb{H}_{d_{1}} \otimes \mathbb{H}_{d_{2}}$ we have the inner product given by $\left\langle f_{1} \otimes g_{1}, f_{2} \otimes g_{2}\right\rangle:=$ $\left\langle f_{1}, f_{2}\right\rangle\left\langle g_{1}, g_{2}\right\rangle$ for each $f_{1} \otimes g_{1}, f_{2} \otimes g_{2} \in \mathbb{H}_{d_{1}} \otimes \mathbb{H}_{d_{2}}$. Here we build BUNTFs as a tensor product of BUNTFs.

Proposition 8.4. Let $\mathcal{F}=\left(f_{k}\right)_{k=1}^{K}$ be a sequence in $\mathbb{H}_{d_{1}}$ and $\mathcal{G}=\left(g_{l}\right)_{l=1}^{L}$ be a sequence in $\mathbb{H}_{d_{2}}$. Then the tensor product $\mathcal{F} \otimes \mathcal{G}:=\left(f_{j} \otimes g_{k}\right)_{k, l=1}^{K, L}$ is a BUNTF for $\mathbb{H}_{d_{1}} \otimes \mathbb{H}_{d_{2}}$ if and only $\mathcal{F}$ is a TF for $\mathbb{H}_{d_{1}}, \mathcal{G}$ is a $T F$ for $\mathbb{H}_{d_{2}}, \mathcal{F}$ or $\mathcal{G}$ is balanced, and $\left\|f_{j}\right\|\left\|g_{k}\right\|=1$ for all $k=1, \ldots, K$, $l=1, \ldots, L$.

Proof. We have $T_{\mathcal{F} \otimes \mathcal{G}}=T_{\mathcal{F}} \otimes T_{\mathcal{G}}$ and $S_{\mathcal{F} \otimes \mathcal{G}}=S_{\mathcal{F}} \otimes S_{\mathcal{G}}$. By [34, Corollary 5.1], $\mathcal{F} \otimes \mathcal{G}$ is a UNTF for $\mathbb{H}_{d_{1}} \otimes \mathbb{H}_{d_{2}}$ if and only $\mathcal{F}$ is a $\mathrm{TF}$ for $\mathbb{H}_{d_{1}}, \mathcal{G}$ is a TF for $\mathbb{H}_{d_{2}}$ and $\left\|f_{j}\right\|\left\|g_{k}\right\|=1$ for all $k=1, \ldots, K$, $l=1, \ldots, L$.

Let $\left(e_{m}\right)_{m=1}^{d_{1}}$ be an orthonormal basis for $\mathbb{H}_{d_{1}}$ and $\left(e_{n}\right)_{n=1}^{d_{2}}$ be an orthonormal basis for $\mathbb{H}_{d_{2}}$. Since $\left\langle T_{\mathcal{F}} \otimes T_{\mathcal{G}}(e), e_{m} \otimes e_{n}\right\rangle=\left\langle T_{\mathcal{F}}(e), e_{m}\right\rangle\left\langle T_{\mathcal{G}}(e), e_{n}\right\rangle$ for each $m=1, \ldots, d_{1}$ and $n=1, \ldots, d_{2}$, $\mathcal{F} \otimes \mathcal{G}$ is balanced if and only if $\mathcal{F}$ or $\mathcal{G}$ is balanced.

8.1. Other constructions. For sequences $\mathcal{F}=\left(f_{k}\right)_{k=1}^{K}, \mathcal{G}=\left(g_{l}\right)_{l=1}^{L}$ in $\mathbb{H}_{d}$ we consider the union $\mathcal{F} \cup \mathcal{G}:=\left(\left(f_{k}\right)_{k=1}^{K},\left(g_{l}\right)_{l=1}^{L}\right)$. In this subsection we introduce other techniques for constructing BUNTFs that combine unions and direct sums. Among them, there are methods that can be applied to obtain the five platonic solids in $\mathbb{R}^{3}$.

The next theorem generalizes the method in 31 for obtaining the vertices of the tetrahedron and of the dodecahedron in $\mathbb{R}^{3}$ starting from the third roots of the unity and from the fifth roots of the unity in a plane, respectively.

Theorem 8.5. Let $\alpha, \beta \in \mathbb{F} \backslash\{0\}$. Assume that $\mathcal{F}=\left(f_{k}\right)_{k=1}^{K}$ is a BUNTF for $\mathbb{H}_{d_{1}}, \mathcal{G}=\left(g_{k}\right)_{k=1}^{K}$ where $g_{k}=h$ with $h \in \mathbb{H}_{d_{2}}$ and $\|h\|=1$ for each $k=1, \ldots, K$. Let $y \in \mathbb{H}_{d_{2}}$ with $\|y\|=1$. Then $(\alpha \mathcal{F} \oplus \beta \mathcal{G}) \cup(0, y)$ is a BUNTF for $\mathbb{H}_{d_{1}} \oplus \mathbb{H}_{d_{2}}$ if and only if $d_{2}=1, y=-h, K=d_{1}+1$, $|\alpha|^{2}=1-\frac{1}{K^{2}}$ and $|\beta|^{2}=\frac{1}{K^{2}}$.

Proof. The sequence $(\alpha \mathcal{F} \oplus \beta \mathcal{G}) \cup(0, y)$ is balanced if and only if $y=-K \beta h$. Since $\|h\|=\|y\|=1$, $\beta=\frac{1}{K}$. Consequently, $y=-h$.

For each $f \in \mathbb{H}_{d_{1}}$ and $g \in \mathbb{H}_{d_{2}}$,

$$
S_{(\alpha \mathcal{F} \oplus \beta \mathcal{G}) \cup(0, y)}(f, g)=\left(|\alpha|^{2} \frac{K}{d_{1}} f+\alpha \bar{\beta} T_{\mathcal{F}} T_{\mathcal{G}}^{*} g, \bar{\alpha} \beta T_{\mathcal{G}} T_{\mathcal{F}}^{*} f+|\beta|^{2} S_{\mathcal{G}}(g)+\langle g, y\rangle y\right) .
$$

Taking into account that $\mathcal{F}$ is balanced, $T_{\mathcal{F}} T_{\mathcal{G}}^{*} g=\sum_{k=1}^{K}\left\langle g, g_{k}\right\rangle f_{k}=\langle g, h\rangle \sum_{k=1}^{K} f_{k}=0$ for each $g \in \mathbb{H}_{d_{2}}$. So, $(\alpha \mathcal{F} \oplus \beta \mathcal{G}) \cup(0, y)$ is a TF for $\mathbb{H}_{d_{1}} \oplus \mathbb{H}_{d_{2}}$ if and only if $|\alpha|^{2} \frac{K}{d_{1}}=\frac{K+1}{d_{1}+d_{2}}$ and $\left(K|\beta|^{2}+1\right)\langle g, h\rangle h=\frac{K+1}{d_{1}+d_{2}} g$ for each $g \in \mathbb{H}_{d_{2}}$. The last condition implies that 0 is the only element orthogonal to $h$, therefore $d_{2}=1$. Consequently, $(\alpha \mathcal{F} \oplus \beta \mathcal{G}) \cup(0, y)$ is a TF for $\mathbb{H}_{d_{1}} \oplus \mathbb{H}_{d_{2}}$ if and only if $|\alpha|^{2}=\frac{d_{1}(K+1)}{K\left(d_{1}+1\right)}$ and $\left(K|\beta|^{2}+1\right)=\frac{K+1}{d_{1}+1}$, i.e., $|\beta|^{2}=\frac{K-d_{1}}{K\left(d_{1}+1\right)}$. The two expressions for $|\beta|^{2}$ must be the same, i.e., $\frac{K-d_{1}}{K\left(d_{1}+1\right)}=\frac{1}{K^{2}}$, and this is equivalent to $K=1$ or $K=d_{1}+1$. The first case cannot happen because $\mathcal{F}$ is balanced. So, $K=d_{1}+1$.

Since $\mathcal{F}$ is UN, $\|h\|=\|y\|=1$ and $|\alpha|^{2}+|\beta|^{2}=1$, we have that $(\alpha \mathcal{F} \oplus \beta \mathcal{G}) \cup(0, y)$ is UN.

The proofs of the following results use arguments similar to the previous ones, so we omit them.

The vertices of the octahedron in $\mathbb{R}^{3}$ form a BUNTF that can be obtained adding orthogonally two antipodal points to the BUNTF consisting of the 4th-roots of unity in a plane (see 31]). The next theorem generalizes this construction to an arbitrary direct sum of two Hilbert spaces. Let a BUNTF for $\mathbb{H}_{d_{1}}$ be immersed in a direct sum $\mathbb{H}_{d_{1}} \oplus \mathbb{H}_{d_{2}}$, and add to it one unit-norm vector of $\mathbb{H}_{d_{1}} \oplus \mathbb{H}_{d_{2}}$ and its opposite. We show that the resulting set is a BUNTF for $\mathbb{H}_{d_{1}} \oplus \mathbb{H}_{d_{2}}$ if and only if $\mathbb{H}_{d_{2}}$ is 1 -dimensional, and the added vector is orthogonal to the elements of the given frame in $\mathbb{H}_{d_{1}} \oplus \mathbb{H}_{d_{2}}$. 
Theorem 8.6. Let $\mathcal{F}=\left(f_{k}\right)_{k=1}^{K}$ be a BUNTF for $\mathbb{H}_{d_{1}}, x \in \mathbb{H}_{d_{1}}$ and $y \in \mathbb{H}_{d_{2}}, y \neq 0$. Then $\left(\left(f_{k}, 0\right)\right)_{k=1}^{K} \cup(x, y) \cup(-x,-y)$ is a BUNTF for $\mathbb{H}_{d_{1}} \oplus \mathbb{H}_{d_{2}}$ if and only if $x=0$, $d_{2}=1$, $\|y\|=1$ and $K=2 d_{1}$.

The procedure of the following theorem can be thought as a kind of symmetric simple lift (see [34. Definition 5.2] for the notion of lift and simple lift). It also can be seen as a generalization of the procedure used in 31 for obtaining the vertices of the hexahedron and of the dodecahedron in $\mathbb{R}^{3}$ starting from the BUNTFs in a plane consisting of the fourth roots of the unity and of the fifth roots of the unity, respectively.

Theorem 8.7. Let $\alpha \in \mathbb{F} \backslash\{0\}$. Let $\mathcal{F}=\left(f_{k}\right)_{k=1}^{K}, \mathcal{G}=\left(g_{k}\right)_{k=1}^{K}$ be BUNTFs for $\mathbb{H}_{d}$ and $\mathcal{H}_{k}=$ $\left(\beta_{k} h_{l}\right)_{k=1}^{K}$ where $\beta_{k} \in \mathbb{F}$ for each $k=1, \ldots, K, h_{l} \in \mathbb{H}_{1, l},\left\|h_{l}\right\|=1$ and $\operatorname{dim}\left(\mathbb{H}_{1, l}\right)=1$ for all $l=1, \ldots, r$. Then $\left(\alpha \mathcal{F} \oplus \mathcal{H}_{1} \oplus \ldots \oplus \mathcal{H}_{r}\right) \cup\left(\alpha \mathcal{G} \oplus\left(-\mathcal{H}_{1}\right) \oplus \ldots \oplus\left(-\mathcal{H}_{r}\right)\right)$ is a BUNTF for $\mathbb{H}_{d} \oplus \mathbb{H}_{1,1} \oplus \ldots \oplus \mathbb{H}_{1, r}$ if and only if $r=1, \sum_{k=1}^{K} \overline{\beta_{k}} f_{k}=\sum_{k=1}^{K} \overline{\beta_{k}} g_{k},|\alpha|^{2}=\frac{d}{d+1}$ and $\left|\beta_{k}\right|^{2}=\frac{1}{d+1}$ for each $k=1, \ldots, K$.

Note that one choice for $\beta_{k}$ in Theorem 8.7 is $\beta_{k}=\sqrt{\frac{1}{d+1}}$ for each $k=1, \ldots, K$.

The next method to construct UNTFs can be seen as a partial simple lift.

Proposition 8.8. Assume $\alpha, \beta \in \mathbb{F} \backslash\{0\}$ and $L d_{1}>K$. Let $\mathcal{F}=\left(f_{k}\right)_{k=1}^{K}, \mathcal{G}=\left(g_{l}\right)_{l=1}^{L}$ be a UNTF and a BUNTF for $\mathbb{H}_{d_{1}}$, respectively. Let $\mathcal{H}=\left(h_{l}\right)_{l=1}^{L}$ where $h_{l}=h \in \mathbb{H}_{d_{2}}$ and $\|h\|=1$ for each $l=1, \ldots, L$. Then $\left(\left(f_{k}, 0\right)\right)_{k=1}^{K} \cup(\alpha \mathcal{G} \oplus \beta \mathcal{H})$ is a UNTF for $\mathbb{H}_{d_{1}} \oplus \mathbb{H}_{d_{2}}$ if and only if $d_{2}=1$, $|\alpha|^{2}=\frac{d_{1} L-K}{\left(d_{1}+1\right) L}$ and $|\beta|^{2}=\frac{K+L}{\left(d_{1}+1\right) L}$.

Now we consider a variation of the previous method for obtaining a BUNTF. It can be seen as a symmetric partial simple lift.

Theorem 8.9. Assume $\alpha, \beta \in \mathbb{F} \backslash\{0\}$ and $K<2 d_{1} L$. Let $\mathcal{F}=\left(f_{k}\right)_{k=1}^{K}, \mathcal{G}=\left(g_{l}\right)_{l=1}^{L}$ and $\widetilde{\mathcal{G}}=\left(\widetilde{g}_{l}\right)_{l=1}^{L}$ be BUNTFs for $\mathbb{H}_{d_{1}}$. Let $\mathcal{H}=\left(h_{l}\right)_{l=1}^{L}$ where $h_{l}=h \in \mathbb{H}_{d_{2}}$ and $\|h\|=1$ for each $l=1, \ldots, L$. Then $\left(\left(f_{k}, 0\right)\right)_{k=1}^{K} \cup(\alpha \mathcal{G} \oplus \beta \mathcal{H}) \cup(\alpha \widetilde{\mathcal{G}} \oplus(-\beta \mathcal{H}))$ is a BUNTF for $\mathbb{H}_{d_{1}} \oplus \mathbb{H}_{d_{2}}$ if and only if $T_{\mathcal{G}} e=T_{\widetilde{\mathcal{G}}} e, d_{2}=1,|\alpha|^{2}=\frac{2 d_{1} L-K}{2\left(d_{1}+1\right) L}$ and $|\beta|^{2}=\frac{2 L+K}{2\left(d_{1}+1\right) L}$.

The following theorem generalizes [2, Theorem 3], which is about $t$-designs in $\mathbb{R}^{3}$, for the case $t=2$.

Theorem 8.10. Assume $\alpha_{m}, \beta_{m} \in \mathbb{F} \backslash\{0\}$ such that $\left|\alpha_{m}\right|^{2}+\left|\beta_{m}\right|^{2}=1$ for each $m=1, \ldots, M$. Let $\mathcal{F}_{m}=\left(f_{m, k}\right)_{k=1}^{K}$ be BUNTFs for $\mathbb{H}_{d_{1}}$ for each $m=1, \ldots, M$. Let $\mathcal{G}=\left(g_{k}\right)_{k=1}^{K}$ where $g_{k}=g \in \mathbb{H}_{d_{2}}$ and $\|g\|=1$ for each $k=1, \ldots, K$. Then $\bigcup_{m=1}^{M}\left(\alpha_{m} \mathcal{F}_{m} \oplus \beta_{m} \mathcal{G}\right)$ is a BUNTF for $\mathbb{H}_{d_{1}} \oplus \mathbb{H}_{d_{2}}$ if and only if $d_{2}=1, \sum_{m=1}^{M} \beta_{m}=0$ and $\sum_{m=1}^{M}\left|\beta_{m}\right|^{2}=\frac{|M|}{d_{1}+1}$.

Remark 8.11. An example of scalars $\beta_{m}$ as in Theorem 8.10 is $\beta_{m}=\sqrt{\frac{|M|}{c\left(d_{1}+1\right)}} \widetilde{\beta}_{m}$ where $\widetilde{\beta}_{m} \in \mathbb{F}$, $\frac{1}{|M|} \leq\left|\widetilde{\beta}_{m}\right|^{2} \leq \frac{d_{1}+1}{|M|}$ for each $m=1, \ldots, M, \sum_{m=1}^{M} \widetilde{\beta}_{m}=0$ and $c=\sum_{m=1}^{M}\left|\widetilde{\beta}_{m}\right|^{2}$. Another option is to consider any row of $T_{\mathcal{F}}$ where $\mathcal{F}$ is a BTF for $\mathbb{F}^{d_{1}+1}$ with $M$ elements.

Remark 8.12. Observe that we can vary $\mathcal{F}, \mathcal{G}, \mathcal{H}$, etc., in all the above constructions obtaining in this manner an infinite number of non unitary equivalent BUNTFs. We can also combine these methods generating a great variety of them.

We note that there exist BUNTFs of $K$ points for $\mathbb{R}^{d}$ with $K \geq 2$ unless $K \leq d$ or $K=d+2$ and $K$ is odd. This is a consequence of Proposition 3.5 and results of [26]. 


\section{ACKNOWLEDGEMENT}

This research has been supported by Grants PIP 112-201501-00589-CO (CONICET), PROICO 03-1618 (UNSL), PICT-2014-1480 and UBACyT 20020130100422BA. We thank the anonymous referee for valuable comments that helped to improve the paper.

\section{REFERENCES}

[1] Alexeev B., Cahill J., Mixon D.G.: Full spark frames. J. Fourier Anal. Appl. 18, 1167-1194 (2012).

[2] Bajnok B.: Construction of designs on the 2-sphere. European J. Combin. 12, 377-382 (1991).

[3] Bannai E., Bannai E.: A survey on spherical designs and algebraic combinatorics on spheres. European J. Combin. 30(6), 1392-1425 (2009).

[4] Barg A., Glazyrin A., Okoudjou K., Yu W.-H.: Finite two-distance tight frames. Linear Algebra Appl. 475, 163-175 (2015).

[5] Benedetto J.J., Fickus M. : Finite normalized tight frames. Adv. Comput. Math. 18(2-4), 357-385 (2003).

[6] Benedetto J., Yilmaz Ö., Powell A.: Sigma-delta quantization and finite frames. In: Proc. IEEE International Conference on Acoustics, Speech, and Signal Processing, vol. 3, ICASSP'04, IEEE, Philadelphia, PA, pp. 937-940 (2004).

[7] Bodmann B.G., Paulsen V.I.: Frame paths and error bounds for sigma-delta quantization. Appl. Comp. Harm. Anal. 22, 176-197 (2007).

[8] Bodmann B.G., Paulsen V.I.: Optimal frames for erasures. Linear Algebra Appl. 377, 31-51 (2004).

[9] Cahill J.: Flags, frames, and Bergman spaces. M.S. Thesis, San Francisco State University (2010).

[10] Casazza P.G., Kovačević J.: Equal norm tight frames with erasures. Adv. Comput. Math. 18(2-4), 387-430 (2003).

[11] Casazza P.G., Kutyniok G. Eds.: Finite Frames. Theory and Applications, Birkhäuser, Boston (2012).

[12] Christensen O.: An Introduction to Frames and Riesz Bases, second ed.. Birkhäuser, Boston (2016).

[13] Copenhaver M.S., Kim Y.H., Logan C., Mayfield K., Narayan S.K., Petro M.J., Sheperd J.: Diagram vectors and tight frame scaling in finite dimensions. Oper. Matrices 8(1), 73-88 (2014).

[14] Cox I.J., Kilian J., Leighton T., Shamoon T.: Secure spread spectrum watermarking for multimedia. IEEE Trans. Image Processing 6(12), 1837-1687 (1997).

[15] Coxeter H.S.M.: Regular Polytopes, third ed.. Dover Publications Inc., New York (1973).

[16] Goyal V.K., Kovačević J., Kelner J.A.: Quantized frame expansions with erasures. Appl. Comp. Harm. Anal. 10, 203-233 (2001).

[17] Jimenez D., Wang L., Wang Y.: White noise hypothesis for uniform quantization errors. SIAM J. Math. Anal. 38, 2042-2056 (2007).

[18] Fickus M., Mixon D.G., Tremain J.C.: Steiner equiangular tight frames. Linear Algebra Appl. 436, 1014-1027 (2012).

[19] Han D., Kornelson K., Larson D., Weber E.: Frames for under-graduates. Student Mathematical Library, vol. 40, American Mathematical Society, Providence, RI (2007).

[20] Hong Y.: On spherical $t$-designs in $\mathbb{R}^{2}$. Europ. J. Combinatorics 3, 255-258 (1982).

[21] Horadam K.J.: Hadamard Matrices and Their Applications. Princeton University Press, New York (2007).

[22] Horn R.A., Johnson C.R.: Matrix Analysis, second ed.. Cambridge University Press, New York (2013).

[23] Kang X., Huang J., Zeng W.: Improving robustness of quantization-based image watermarking via adaptive receiver. IEEE Trans. on Multimedia 10(6), 953-959 (2008).

[24] Kovačević J., Chebira A.: An introduction to frames. Found. Trends Signal Process. 2, 1-94 (2008).

[25] Lu C.S., Sun S.W., Hsu C.Y., Chang P.C.: Media hash-dependent image watermarking resilient against both geometric attacks and estimate attacks based on false positive-oriented detection. IEEE Trans. on Multimedia $8(4), 668-685$ (2006).

[26] Mimura Y.: A construction of spherical 2-designs. Graphs and Combinatorics 6(4), 369-372 (1990).

[27] Moulin P., Koetter R.: Data hiding codes. Procs. of IEEE 93(12), 2083-2126 (2005).

[28] Murdoch T.A.: Isogonal configurations. Am. Math. Monthly 100(4), 381-384 (1993).

[29] Okoudjou K. Ed.: Finite Frame Theory. A Complete Introduction to Overcompletness. Proc. of Symposia in Applied Mathematics, AMS, Vol. 23 (2016).

[30] Püschel M., Kovačević J.: Real, tight frames with maximal robustness to erasures. Proc. Data Compression Conference Snowbird UT, 63-72 (2005).

[31] Safapour A., Shafiee M.: Constructing finite frames via Platonic solids. Iran. J. Math. Sci. Inform. 7(1), 35-42 (2012). 
[32] Strohmer T., Heath R.W. Jr.: Grassmannian frames with applications to coding and communication. Appl. Comp. Harm. Anal. 14(3), 257-275 (2003).

[33] Sustik M.A., Tropp J.A., Dhillon I.S., Heath R.W. Jr.: On the existence of equiangular tight frames. Linear Algebra Appl. 426, 619-635 (2007).

[34] Waldron S.: An Introduction to Finite Tight Frames. Birkhäuser, Boston (2018). 